\title{
Evaluation of Measurement
}

\section{Uncertainties of DVC applied to}

\section{Laminography Data}

\author{
Ante Buljac, ${ }^{1,2}$ Thibault Taillandier-Thomas, ${ }^{1,2}$ \\ Lukas Helfen, ${ }^{3,4}$ Thilo F. Morgeneyer, ${ }^{2}$ and François Hild ${ }^{1, *}$
}

${ }^{1}$ Laboratoire de Mécanique et Technologie (LMT)

ENS Paris-Saclay, CNRS UMR 8535, Université Paris-Saclay

61 avenue du Président Wilson, 94235 Cachan Cedex, France

*Corresponding author, email: hild@lmt.ens-cachan.fr

${ }^{2}$ MINES ParisTech, PSL Research University, Centre des Matériaux

CNRS UMR 7633, BP 87, 91003 Evry, France

${ }^{3}$ ANKA / Institute for Photon Science and Synchrotron Radiation

Karlsruhe Institute of Technology (KIT), D-76131 Karlsruhe, Germany

${ }^{4}$ European Synchrotron Radiation Facility (ESRF), 38043 Grenoble, France 


\begin{abstract}
The effect of different data acquisition parameters on the displacement and strain uncertainties of kinematic fields is assessed via Digital Volume Correlation (DVC) applied to three-dimensional (3D) data sets imaged by synchrotron laminography. The measurement uncertainty is estimated for i) different materials and associated varying contrast, ii) repeated scans, i.e., uncertainty due to the imaging system and 3D reconstruction iii) rigid body motions between two subsequent scans, iv) rescaling of the gray level histogram during 32-bit floating point to 8-bit integer data conversion, v) changes in the beam properties, i.e., use of a monochromator or an undulator, and vi) changes in camera/detector characteristics. It is found that the amount of image contrast is not the only parameter that controls uncertainties for different materials. Further, the rigid body motion procedure should be preferred over repeated scans as it provides more conservative measurement uncertainty values. The applied 32 to 8 -bit conversion procedure, beam tuning and detector characteristics hardly affect the measurement uncertainty.
\end{abstract}

Keywords: Digital Volume Correlation; Kinematic measurements; Resolution; Synchrotron laminography; Uncertainty quantification.

\title{
1 Introduction
}

Laminography $[10,15]$ can be considered as a 3D imaging technique complementary to computed tomography since it is particularly adapted to twodimensionally extended specimens. Employing synchrotron radiation [11, 12] 
the main advantages offered by laminography are that regions of interest in large plate-like samples can be imaged with high spatial resolutions [12], high energies [20, 28] and using different phase-contrast imaging modalities [14, 2]. In materials science, applications with panel- and sheet-like samples can be investigated in-situ contrary to tomography, where samples close to match stick or rod shapes $[3,27,7]$ are required. The liberty to employ plate- and sheet-like specimens via synchrotron laminography allows more engineeringrelevant loading conditions to be applied, say, during the study of damage initiation and growth inside materials. For instance, the development of damage has been observed for low stress triaxialities ahead of notched samples during ductile tearing [31, 29, 32].

It is however worth noting that the sampling of the 3D Fourier domain of the region of interest is incomplete in laminography [15], which leads to imaging artifacts [48]. These artifacts are assumed to degrade the displacement and strain resolutions when digital volume correlation (DVC) techniques are applied [30]. Laminography can still be considered as a relatively new technique, especially when considering its use for measuring kinematic fields and strain changes inside material microstructures. It should also be noted that the attainable spatial resolution in synchrotron laminography is higher in directions perpendicular to the rotation axis than in the direction parallel to it. One should therefore also expect higher strain measurement uncertainties along the rotation axis direction.

The aim of the present paper is to assess in a systematic manner the displacement and strain uncertainties measured via DVC when considering synchrotron laminography data. There are various ways of estimating 
the uncertainties of correlation techniques. When only one acquisition is available, artificial motions i.e., generally uniform translations are applied to create a new volume that is subsequently registered with the reference volume $[25,39,4,24,17,26]$. This first type of analysis mainly probes the interpolation scheme of the gray levels (GL) to achieve sub-voxel resolutions. The second type of approach also uses only one reference volume but consists of adding noise to create a new volume, which is correlated with its noisefree reference $[22,21]$. The sensitivity to noise is addressed in this type of procedure. To be fully representative the model should incorporate the full covariances associated with radiograph acquisition and 3D reconstruction. Last, the previous two procedures can be applied experimentally [25, 23]. This method consists of analyzing two consecutive scans of the sample in a given loading state (it may also be unloaded) without rigid body motion (called bis in the following) between the two acquisitions or with a deliberately introduced one (called $\mathrm{rbm}$ ). The main advantage is that both sources of error are investigated at the same time for the material of interest. This last method will be adopted in this work.

The standard uncertainties are reported to have levels higher than those observed in standard 2D-DIC techniques when using local or global DVC approaches [25, 24, 23, 26]. For laminography, an additional degradation has been observed [30] for an aluminum alloy with a very low volume fraction of markers. Since laminography is a very recent technique, there are very few results dealing with DVC calculations $[40,30,46,9,32]$. In particular, measurement uncertainties have been evaluated [30] for one given microstructure (AA 2139) that was deemed very difficult for DVC calculations since the vol- 
ume fraction of secondary particles was less than $1 \%$. This microstructure is also considered hereafter in addition to two additional ones. A second class of materials presented in this work is nodular graphite cast iron. The microstructure scales and topology significantly differ from aluminum alloys. Hence, X-ray projections, reconstructed volume contrast and the final conditions for DVC registrations are changed.

In the reported analyses a global approach to DVC is chosen in which the displacement field is based on discretizations made of 8-noded elements (i.e., C8-DVC [39], see Appendix A). The studied materials and the way the laminography data are obtained are first presented. Next, the measurement uncertainties are evaluated for the chosen microstructures for two different cases:

- measurement resolution applied to repeated scans (bis) of the object;

- measurement uncertainty applied to scans of the object moved (rigid body motion - rbm) prior the second acquisition.

Possible relationships between the underlying microstructure, sample geometry, experimental setup and uncertainty levels are discussed. Last, additional effects are analyzed on a more limited amount of scans:

- influence of 32-bit floating point to 8-bit integer data conversion (i.e., rescaling of the gray level histogram);

- influence of the beam properties (i.e., use of monochromator or undulator);

- influence of the employed optical camera/detector. 
The listed items will be detailed in Section 2.3.

\section{Analyzed Materials and Configurations}

Four different materials will be studied herein:

- AA2139 grade (i.e., Al-Cu-Mg alloy), whose intermetallic volume fraction has been determined to be $0.45 \%$ with an initial void volume fraction of $0.34 \%$ (see Figure 1(a)). A feasibility study and analysis was performed in terms of measurement uncertainties [30]. Rather high levels of uncertainties were reported and one question is related to the representativeness of these results with other materials such as those studied hereafter. Samples with two different heat treatments are examined, namely, T3 denotes solution heat-treated, stretched between 2-4\% and naturally aged to obtain the T351 (T3) condition. Conversely, T8 stands for an artificial aging treatment. These heat treatments should not change the image contrast at micrometer scale. The flat to slant transition was studied for this alloy in its T3 state [32].

- AA2198 grade (i.e., Al-Cu-Li alloy), which has no initial porosity and $0.3 \%$ volume fraction of metallic inclusions (see Figure 1(b)). For this grade, the flat to slant transition associated with ductile fracture was also analyzed thanks to DVC measurements [29, 9]. This material has the lowest volume fraction of secondary phases (i.e., particles and voids). Again, two different heat treatments (T3 and T8) will be studied. 
- AA6061 grade (i.e., Al-Mg-Si alloy) is a precipitation hardened aluminum alloy, with $0.25 \%$ volume fraction of coarse $\mathrm{Mg}_{2} \mathrm{Si}$ particles, $0.57 \%$ of iron-rich intermetallics and $0.05 \%$ of porosity (see Figure 1(c)). Compared to Figure 1(a-b), less ring artifacts (i.e., concentric with the specimen rotation center) are apparent. Crack initiation and propagation were investigated for such grade [45] but no DVC analysis was carried out so far. In that work the T6 heat treatment was inspected.

- Commercial nodular graphite cast iron (EN-GJS-400) represents a second class of materials with different microstructural scales (Figure 1(d)). Holes were drilled in the examined plates. In these analyses, an experimental/numerical framework was developed to validate simulations at the microscopic scale $[8,44]$. 


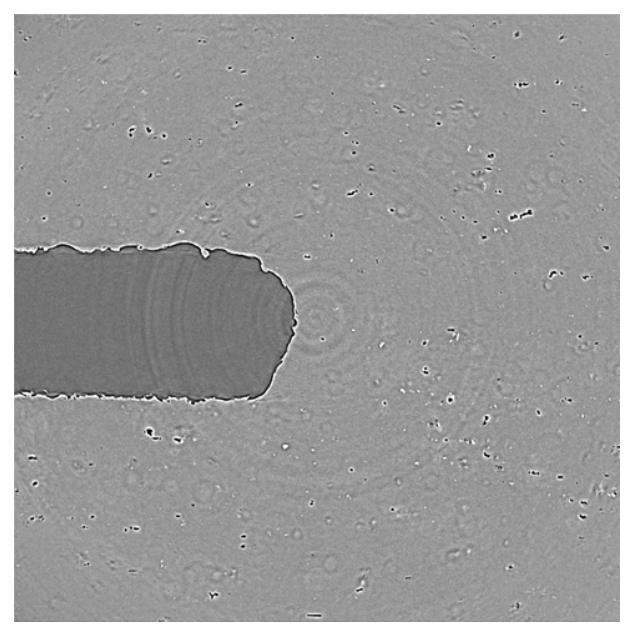

(a) AA2139

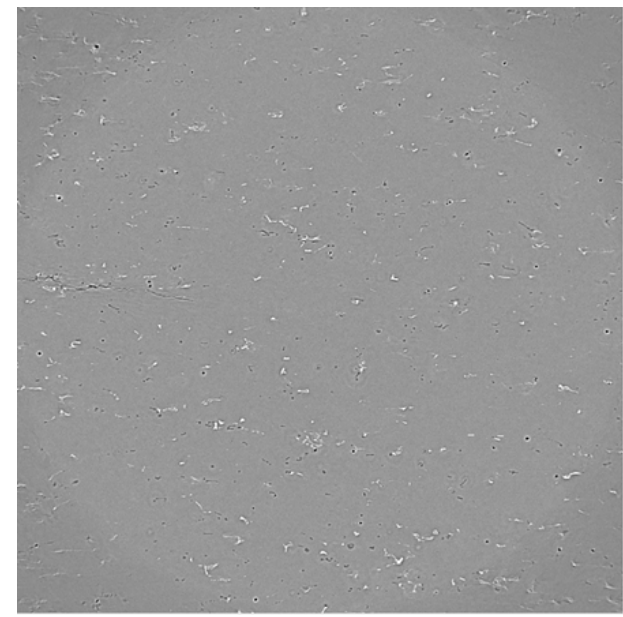

(c) AA6061

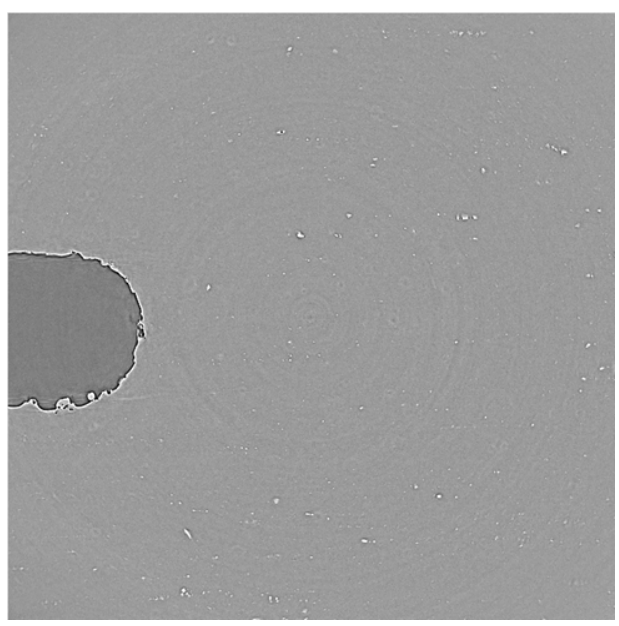

(b) AA2198

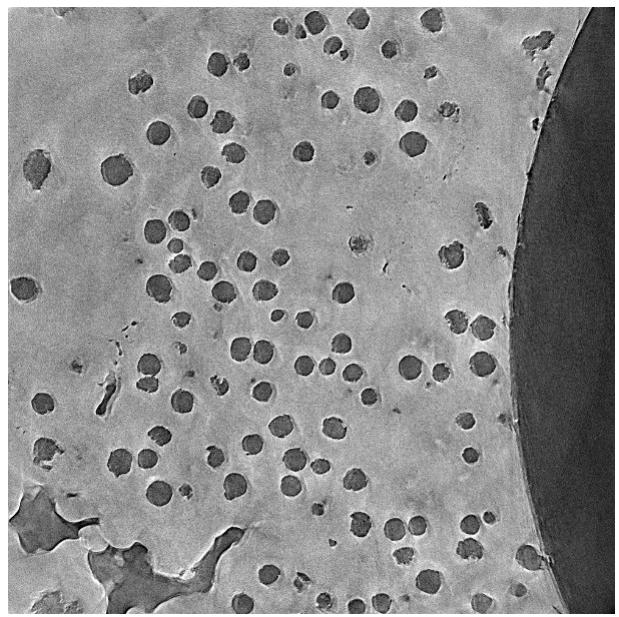

(d) GJS-400

Figure 1: Mid-thickness $L-T$ slice in 3D reconstructed volumes of three different aluminum alloys and one cast iron sample. The picture definition is $2040 \times 2040$ pixels for aluminum alloys and $1600 \times 1600$ pixels for cast irons. The physical size of one voxel is $0.7 \mu \mathrm{m}$ for aluminum alloys and $1.1 \mu \mathrm{m}$ for cast iron. Especially for sub-figures (a) and (b), ring artifacts from static features on the projection radiographs are clearly visible 


\subsection{Aluminum alloys}

The alloy forming directions will define the measurement frame used herein (even for cast iron). The rolling direction is denoted as $\mathrm{L}$, the transverse direction as $\mathrm{T}$, and the short-transverse direction in the through-thickness as $\mathrm{S}$. The listed microstructures and heat treatment variations are characterized through gray level histograms in Figure 2. The gray level distributions confirm the poor texture quality where most of the dynamic range (256 gray levels) is just partially used. Additional (i.e., morphological) characterizations of this first class of materials are reported in Appendix B.

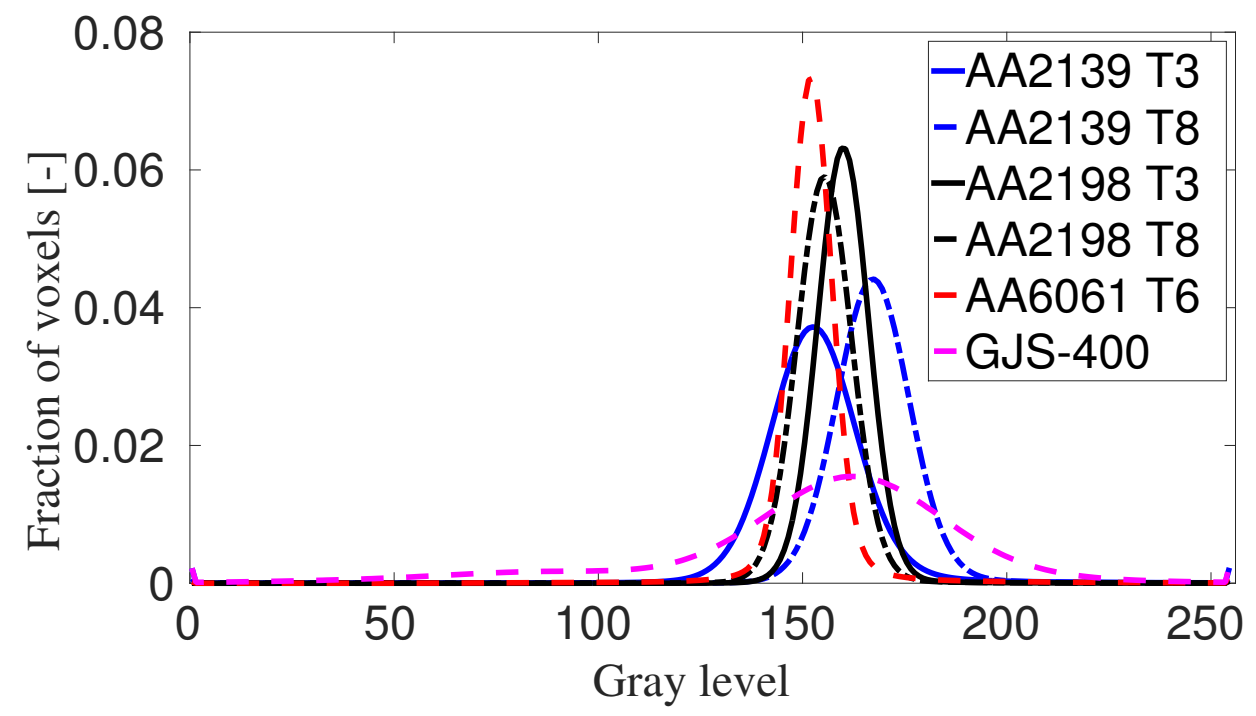

Figure 2: Gray level histograms of the investigated 3D volume images for different aluminum alloys and the cast iron.

The 3D images of aluminum alloys were obtained at the imaging beamline ID19 [33] of the European Synchrotron Radiation Facility (Grenoble, France). An inclination angle of the specimen rotation axis of approximately $25^{\circ}$ with 
respect to the beam normal $\left(\theta \approx 65^{\circ}\right)$ was chosen as well as a monochromatic beam of around $25 \mathrm{keV}$ X-ray energy obtained from a single-bounce multilayer monochromator or a short-period single-harmonic undulator source providing approximately the same monochromaticity. A series of 1500 radiographs is then used to reconstruct 3D volumes by using a filtered-back-projection algorithm [35]. The reconstructed volumes represent the scanned region with a size of $2040 \times 2040 \times 2040$ voxels. The side length of 1 cubic voxel is around $0.7 \mu \mathrm{m}$. Conducting DVC analyses with the full size reconstructed volumes is computationally too demanding and should not be done anyway since the most outward voxels have only information from a limited range of viewing angles (see Appendix C, Figure 20). Therefore, only a part of the reconstructed volume called Region of Interest (ROI) is considered herein.

For aluminum alloys all examined reconstructed volumes belong to compact tension (CT)-like specimen regions ahead of the notch root (AA2139 and AA2198) or crack tip (AA6061). The experimental setup is illustrated in Figure 3. The CT-like specimen has dimensions $60 \times 70 \times 1 \mathrm{~mm}$. It contains either a machined notch of radius $0.17 \mathrm{~mm}$ produced by electrical discharge machining or fatigue pre-cracking has been utilized for AA6061. 


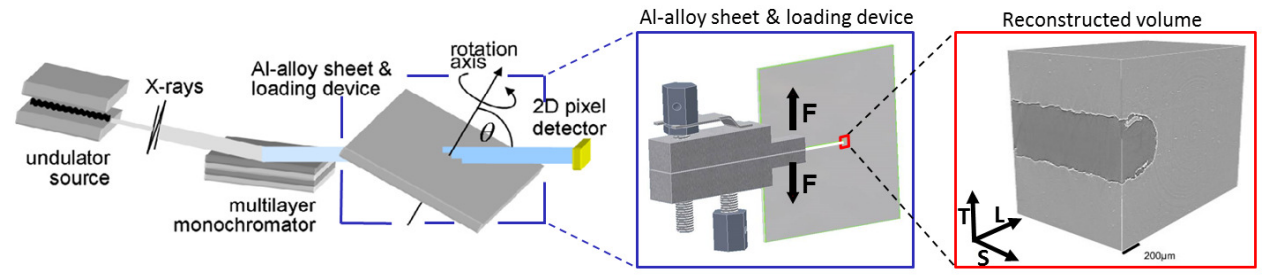

Figure 3: Experimental setup schematically shown for the aluminum CT-like specimens investigated in this work [30]

The first case studied in detail is a CT-like sample made of AA2139 T3. Figure 4(a) shows the location of the Region of Interest (ROI) in the reference and rigid body motion $(r b m)$ configuration moved by $u_{T}=22$ voxels, $u_{L}=1113$ voxels so that the rotation axis and reconstruction artifacts (e.g., rings) are positioned differently in the inspected region. Its size is $1184 \times 512 \times 992$ voxels. All examined ROIs for aluminum alloys have the same size eliminating the potential ROI size effects on the calculated uncertainties. Also, ROIs are positioned roughly at the same location $\approx 800 \mu \mathrm{m}$ from the notch root/tip, whenever available original and $r b m$ scans allowed for it.

The second case is a CT-like sample made of AA2139 T8. Figure 4(b) shows the location of the Region of Interest (ROI) in the reference and rbm configuration moved by $u_{T}=15$ voxels, $u_{L}=595$ voxels and $u_{S}=3$ voxels. 


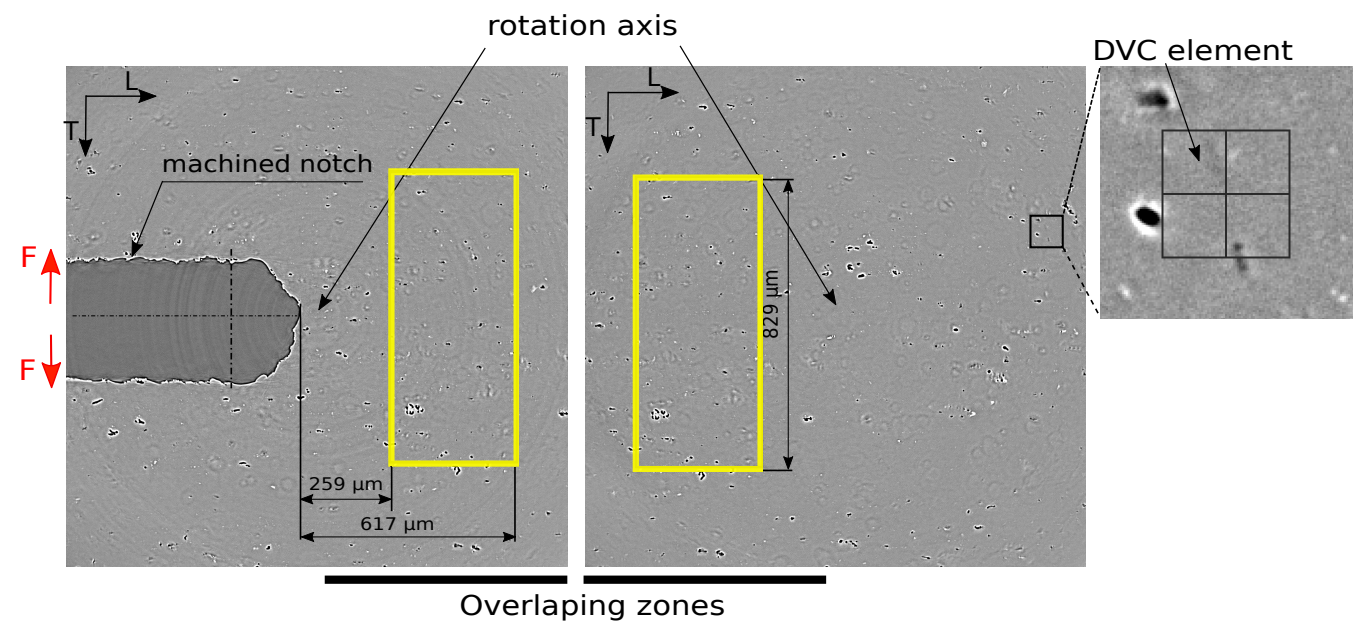

(a) AA2139 T3

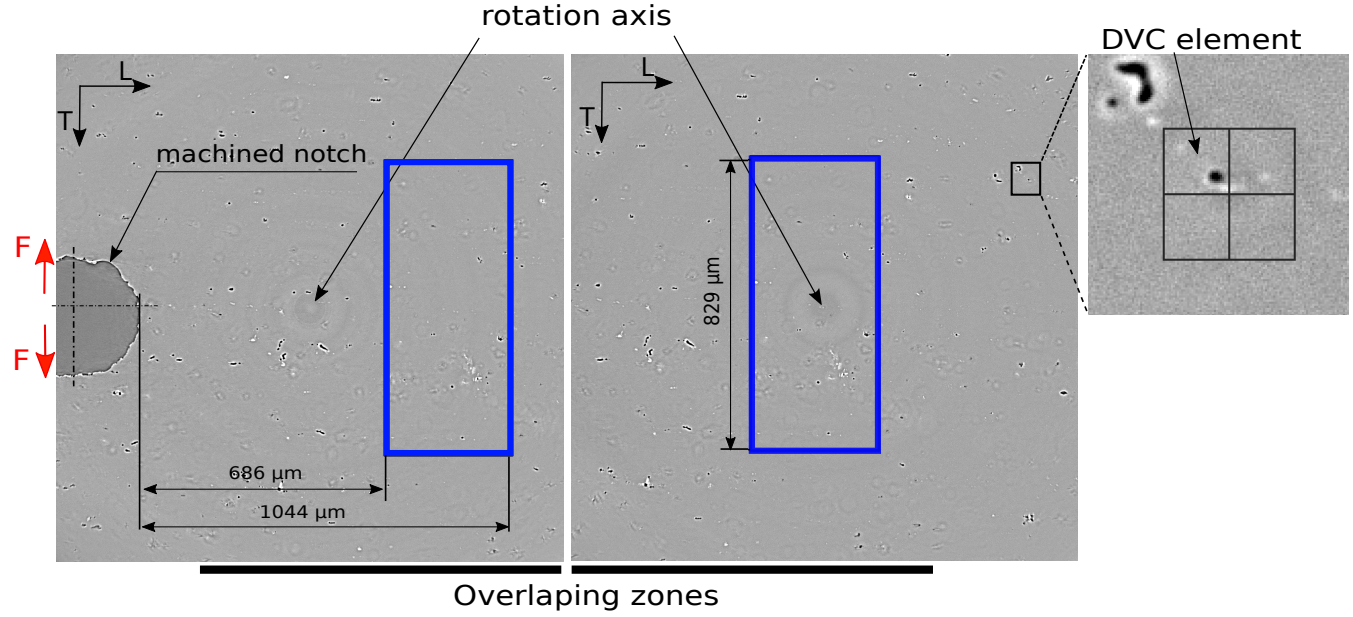

(b) AA2139 T8

Figure 4: Region of interest considered in the uncertainty analysis of the volume made of (a) AA2139 T3 and (b) AA2139 T8. The squares on the right denote 4 DVC elements with isotropic size $\ell=32$ voxels

The third case is a CT-like sample made of AA2198 T3. Figure 5(a) shows the location of the Region of Interest (ROI) in the reference and rbm configuration moved by $u_{T}=-15$ voxels, $u_{L}=586$ voxels and $u_{S}=3$ voxels. The rotation axis and reconstruction artifacts (e.g., rings) are visible. 
The fourth case is a CT-like sample AA 2198 T8. Figure 5(b) shows the location of the Region of Interest (ROI) in the reference and rbm configuration moved by $u_{T}=0$ voxel, $u_{L}=0$ voxel and $u_{S}=45$ voxels. Since there is a small rigid body motion in the $\mathrm{S}$ direction, the rotation axis and reconstruction artifacts are positioned almost identically in the inspected ROI for both scans. 


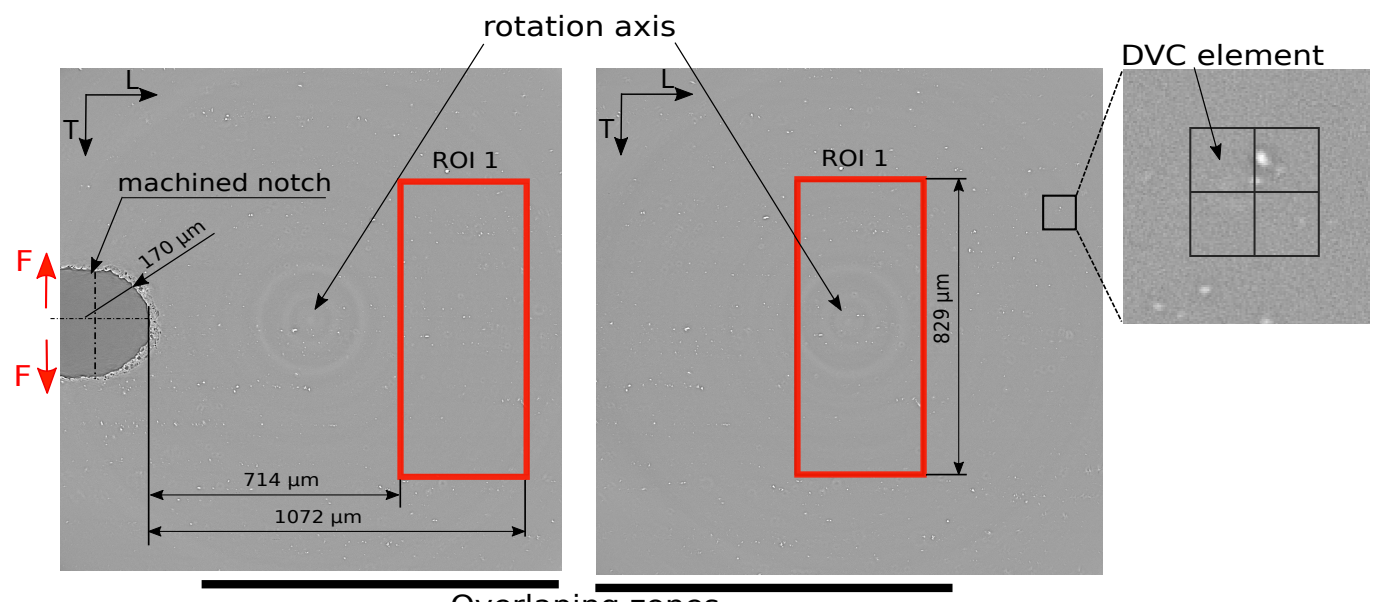

(a) AA2198 T3

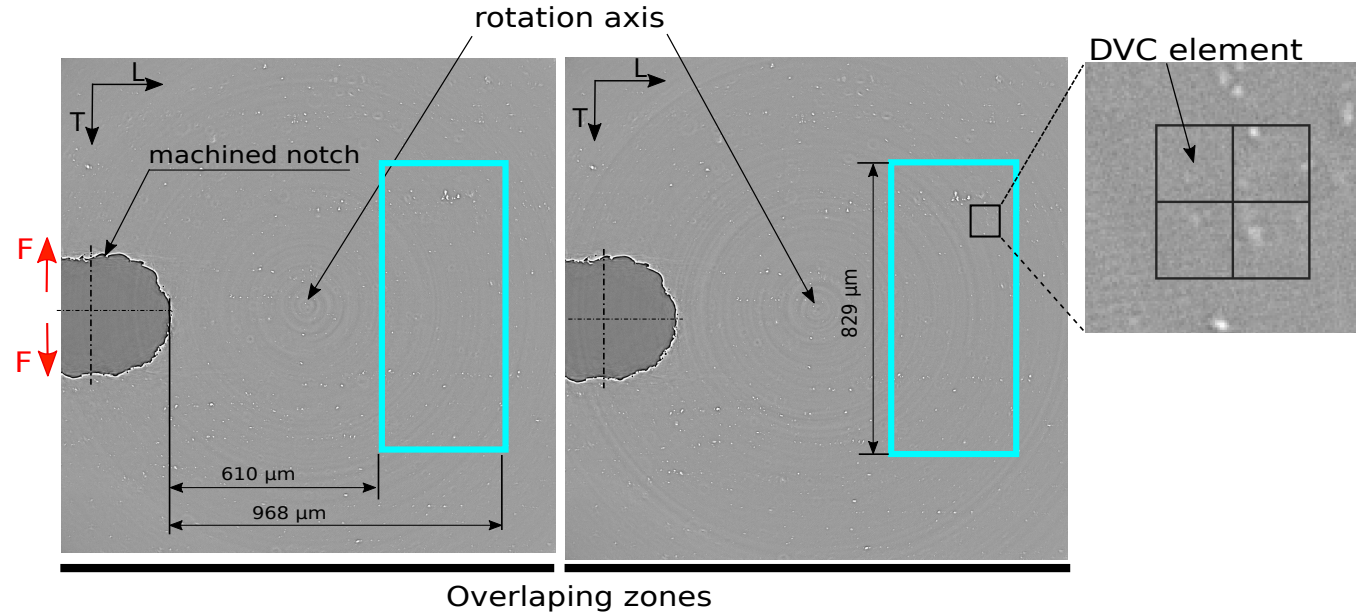

(b) AA2198 T8

Figure 5: Region of interest considered in the uncertainty analysis of the volume made of (a) AA2198 T3 and (b) AA2198 T8. The squares on the right denote 4 DVC elements with isotropic size $\ell=32$ voxels

The fifth case is a CT-like sample made of AA6061 T6. A mechanical shift such that $u_{T}=0, u_{L}=1330$ voxels and $u_{S}=170$ voxels has been performed between the two scans as shown in Figure 6 where the ROI position is depicted. The rotation axis is positioned differently in the inspected ROI 
while reconstruction artifacts are not detected.

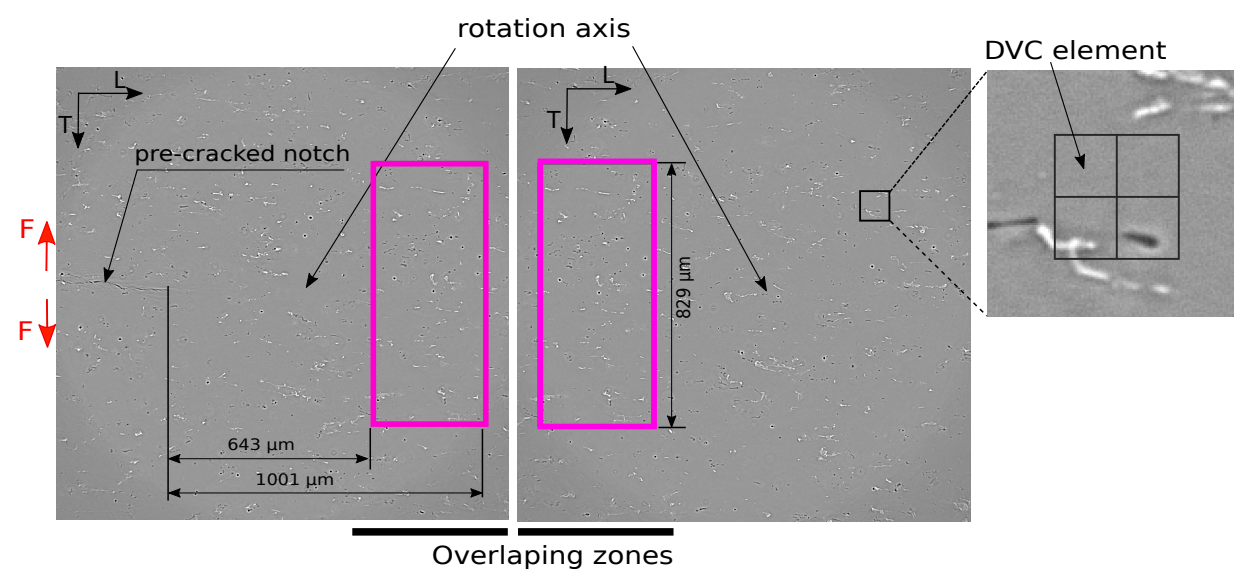

Figure 6: Region of interest considered in the uncertainty analysis of the volume made of AA6061 T6. The squares on the right denote 4 DVC elements with isotropic size $\ell=32$ voxels

\subsection{Nodular graphite cast iron}

Commercial nodular graphite cast iron (serial code EN-GJS-400) is also analyzed. This material has a broader gray level distribution due to the presence of two highly contrasted materials (i.e., ferrite and graphite, see Figures 1 and 2). Additional (i.e., morphological) characterizations of cast iron are reported in Appendix D. It is believed that such microstructure is a priori more favorable to DVC analyses. For the cast iron grade considered herein two tensile samples with different geometries will be examined. First, a plate with a central hole that localizes stresses in the surrounding ligaments is analyzed (Figure 7). One of the ligaments has been scanned during the experiment as shown in Figure 7. The inspected region has a size of $512 \times 512 \times 512$ voxels. The results from this ROI have been used to drive micromechanical simula- 
tions [8]. The position of the ROI in the global coordinate system is shown in Figure 7 while in the through-thickness direction $(\mathrm{S})$ the ROI and sample mid-thickness planes coincide. No rbm scans were performed with this sample geometry.

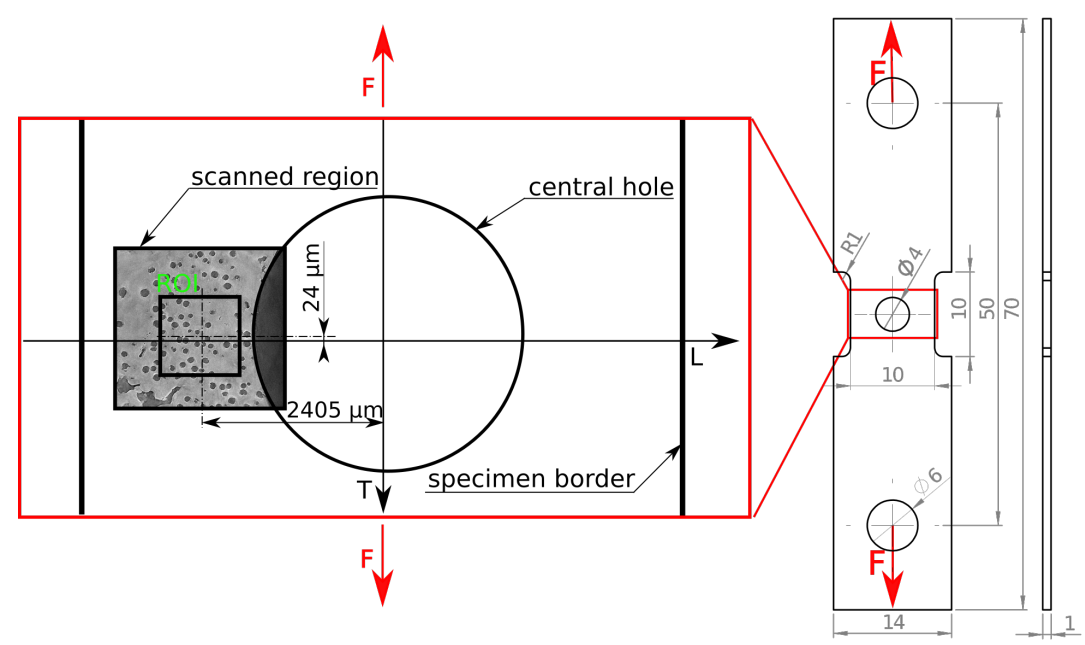

Figure 7: Schematic view of the sample with the scanned region close to the central hole. Section of the reconstructed volume with the position of the region of interest $(\mathrm{ROI})$

The 3D images of cast iron were obtained at beamline ID15A [34] of the European Synchrotron Radiation Facility (Grenoble, France) with a white beam from an in-vacuum undulator source fully closed to $6.25 \mathrm{~mm}$ gap and filtered with 7-mm Si and 1.1-mm Cu attenuators to around $60 \mathrm{keV}$ average energy. For each scan, 4000 projections were acquired with an exposure time of about $5 \mathrm{~ms}$ using an indirect $\mathrm{x}$-ray detector consisting of a freestanding $25-\mu$ m thick LuAG crystal, a $10 \times$ optical magnification and a fast CMOS-based camera with on-camera memory storage of the projection data. 
Each scan took about 200 s. The minimum specimen to detector distance is limited by the detector geometry to about $70 \mathrm{~mm}$. The resulting strongly contrasted edges are also expected to contribute to laminography artifacts typical for incomplete sampling. The reconstructed volume has a size of $1600 \times 1600 \times 1600$ voxels. The side length of 1 voxel is equal to $1.1 \mu \mathrm{m}$.

The second geometry, which is inspired by the work of Weck et al. [47], is shown in Figure 8. The scanned region incorporates two holes while the ROI employed in DVC calculations is mainly concentrated in the ligament between the holes. Figure 8 shows the location of the Region of Interest (ROI) in the reference configuration. The $r b m$ configuration is moved by $u_{T}=$ 68 voxels and $u_{L}=267$ voxels. The results from this ROI have been used to outline all the advantages of using measured DVC boundary conditions when running micromechanical numerical simulations [44]. To be able to keep large ROI sizes, the original reconstructed volumes are a priori coarsened, each 8 neighboring voxels are averaged to form one supervoxel (i.e., binning of $2 \times 2 \times 2$ ). In the following, no distinction will be made between this coarsened configuration and the other ones and the term voxel will still be used irrespective of such operation. 


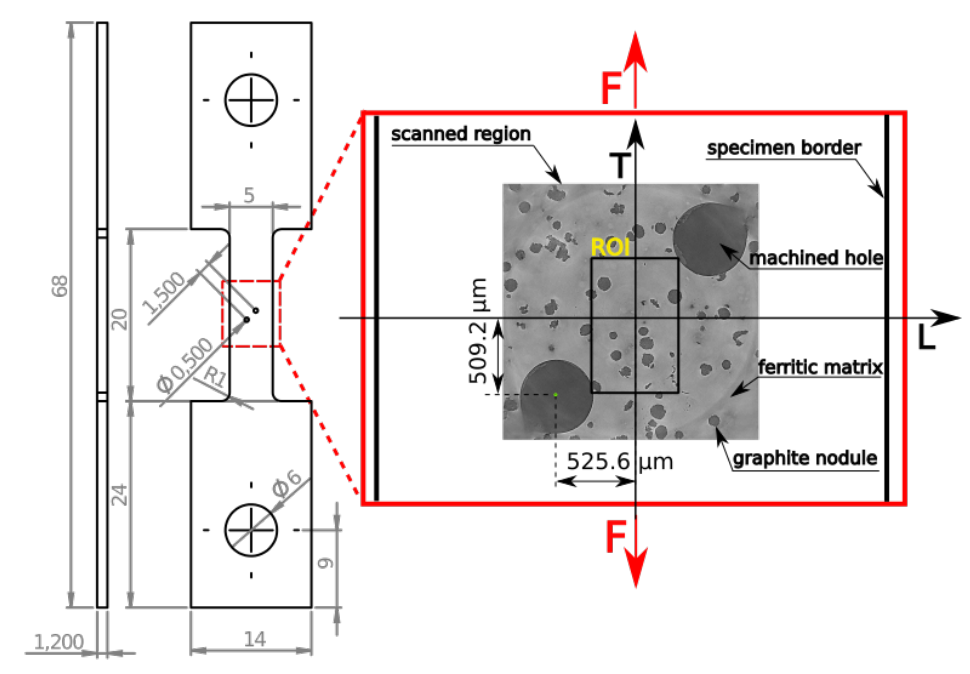

Figure 8: The sample geometry with the scanned region between the two holes. Section of the reconstructed volume with ROI position

\subsection{Procedures for Uncertainty Quantification}

Five different procedures will be applied to analyze the displacement and strain uncertainties of DVC applied to laminography data:

- repeated scan - bis: two scans are acquired without any motion between the two acquisitions. This procedure is standard when aiming for the resolution of the measurement technique [1].

- rigid body motion - rbm: two scans are acquired when a rigid body motion is applied prior to the second acquisition. This case is deemed difficult [30] because the reconstruction artifacts (e.g., rings) do not follow the motion and may severely bias the displacement measurements with low contrasted images as studied herein (Figure 2). To assess the contribution induced by rigid body motions bis and rbm cases are 
compared.

- 32-bit floating point to 8-bit integer conversion: the reconstruction of $3 \mathrm{D}$ volumes from radiographs involves at one stage to map the 32bit floating point values onto 8-bit integer digitizations (i.e., rescaling) given the high level of noise. The transformation is performed by choosing the gray level range for the data to be rescaled. The same gray level range is generally applied for conversion of a series of in situ scans. This may depart from the gray level conservation, which is the underlying principle of DVC (see Equations (3) and (4)). The influence of different rescaling parameters is studied in the case of the AA2139 T3 sample.

- changes in the beam properties: since the data originate from different experimental sessions, the influence of the experimental setup, particulary the monochromator, is also evaluated. Experimental data available for this analysis are from AA2139 T3 and AA2198 T8 alloys observed with a monochromator opposed to AA2139 T8 and AA2198 T3 cases scanned without monochromator but using X-rays from a singleharmonic undulator.

- detector characteristics: the influence of optical camera/detector is also probed. The scans available for AA2198 T8 alloy are sampled with two different cameras.

The overview of listed procedures and cases in which they will be studied are summarized in Table 1. 
Table 1: Uncertainty assessment: procedures and available data

\begin{tabular}{|c|cccccc|}
\hline material & AA2139 T3 & AA2139 T8 & AA2198 T3 & AA2198 T8 & AA6061 T6 & GJS-400 \\
\hline repeated scan - $b i s$ & & $\sqrt{ }$ & $\sqrt{ }$ & $\sqrt{ }$ & $\sqrt{ }$ \\
rigid body motion - rbm & $\sqrt{ }$ & $\sqrt{ }$ & $\sqrt{ }$ & $\sqrt{ }$ & $\sqrt{ }$ \\
32 to 8 bit conversion - rescaling & $\sqrt{ }$ & & $\sqrt{ }$ & $\sqrt{ }$ & & \\
beam properties & $\sqrt{ }$ & $\sqrt{ }$ & $\sqrt{ }$ & & \\
detector influence & & & & $\sqrt{ }$ & \\
\hline
\end{tabular}

All the previous cases are analyzed as follows. DVC analyses are run to measure displacement fields. The latter ones are interpolated to account for rigid body motions (i.e., translations and rotations). The difference between a linear interpolation and measured fields is an estimation of the measurement errors [23]. The root mean square error associated with each displacement component is obtained. From that quantity, the displacement uncertainty $\sigma_{u}$ corresponds to the mean of these three values. Eight-noded cubes are chosen in the finite-element based DVC code (i.e., C8-DVC [39], see Appendix A). The size $\ell$ of each C8 element is the length expressed in voxels of any edge. Different sizes are considered, namely, 16, 24, 32, 48 and 64-voxel elements. However for one case (AA2139 T8) calculations could not be performed with 16-voxel elements because of divergence.

The logarithmic (or Hencky) strains are evaluated by using the mean value of the displacement gradient tensor in each C8 element [18]. The reason for that choice is to account for possible large rotations, even though very small stretches are expected in the following. In the present case, von Mises' equivalent strain $\epsilon_{e q}$ (to assess plastic strains $[29,9,32]$ ) is reported. The reason for this choice will become clearer in the sequel since the level of uncertainties will generally be higher than elastic strains in the studied 
aluminum alloys. Such small uncertainties can only be achieved by considering regularized DVC [22, 21, 46], which is not used herein. Further, it has been checked that the mean strain error was at least one order of magnitude less than the corresponding standard deviation $\sigma_{\epsilon}$. Only the latter will be reported hereafter.

\section{Uncertainty quantifications}

\subsection{Repeated scan - bis case}

For bis cases no rigid body motions are carried out between the two acquired scans so that the effect of noise associated with laminography, reconstruction and DVC will be probed. For this analysis the experimental data are from AA2139 T8, AA2198 T3, AA6061, and cast iron samples (Table 1). For cast

iron, two repeated scans (i.e., bis cases) are available for the central hole experiment (Figure 7), namely, " $0 \mathrm{~mm}$ " in the undeformed state and one more in a deformed state (i.e., "0.744 mm") for the applied displacement. For the two-hole experiment as for all aluminum alloys, only the reference configuration is used for uncertainty quantifications.

Figure $9(\mathrm{a}-\mathrm{c})$ shows the standard displacement uncertainties along the three directions as functions of the element size for all studied configurations. There is no clear effect of the three directions on the level of displacement uncertainty. This observation is confirmed when each component uncertainty is compared with the mean value reported in Figure 9(d). Compared with earlier results [30], the present uncertainties are lower and there is no clear 
difference between the direction along the rotation axis and those in the perpendicular plane. One of the reasons is that in present case, no motions are applied between the two acquisitions. This effect will be investigated in Section 3.2.

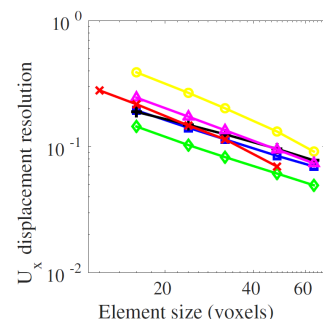

(a) $\mathrm{T}$

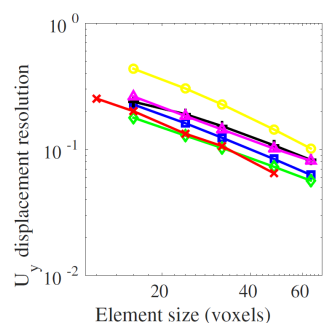

(b) $\mathrm{L}$

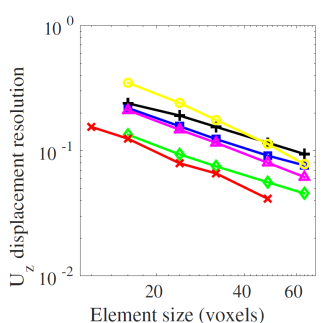

(c) $\mathrm{S}$

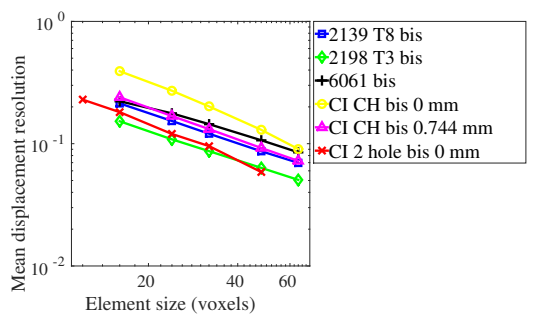

(d) mean

Figure 9: Standard displacement resolutions as functions of the element size for bis cases

It has been shown [21] that the resolution of correlation techniques is proportional to the inverse of the DVC matrix $[\mathbf{M}]$ (see Equation (7) in Appendix A) multiplied by twice the variance of noise and the physical size of the pixel/voxel when controlled by acquisition noise. This means that depending on the gradient (i.e., contrast) of the underlying microstructure the resolution of correlation technique is not necessarily uniform over the ROI. Figure 10 shows the 100 largest eigenvalues of the $[\mathbf{M}]^{-1}$ matrix for the studied microstructures and element size $\ell=32$ voxels. This graph shows 
very isolated influences of microstructure quality.

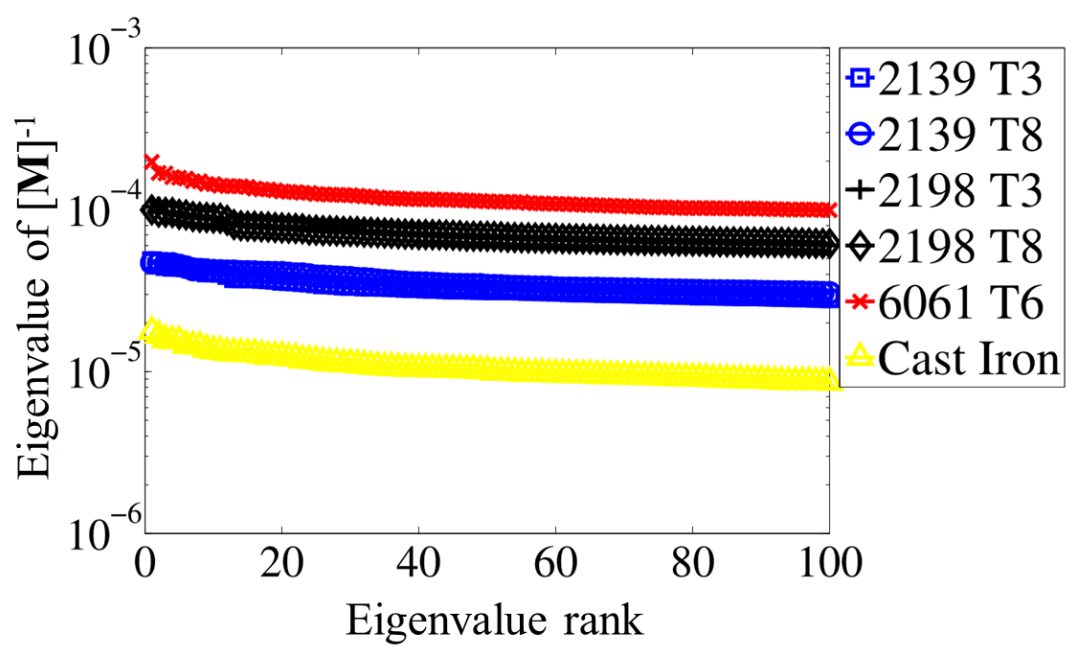

Figure 10: The largest 100 eigenvalues of the inverse DVC matrix $[\mathbf{M}]^{-1}$ for the studied microstructures and element size $\ell=32$ voxels

From Figure 9(d), which does not show the same relative trend (e.g., cast iron should have the lowest uncertainty and AA6061 the highest level, see Figure 10), it is concluded that the quality of the microstructure has a very small influence on the displacement uncertainty level. This trend is confirmed in Figure 11 where the mean displacement uncertainties are recalled and the corresponding standard equivalent strain uncertainty is reported as a function of the element size for all studied configurations. There is virtually no difference for all cases analyzed. 


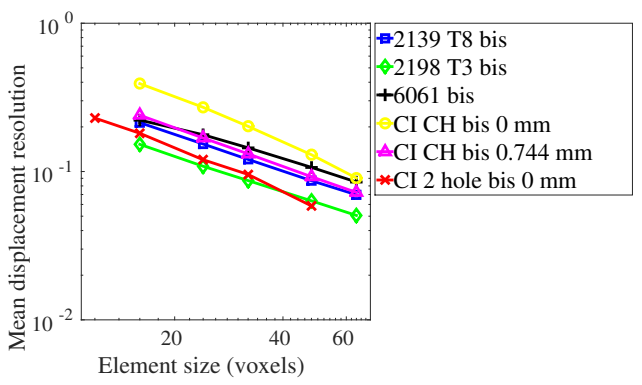

(a)

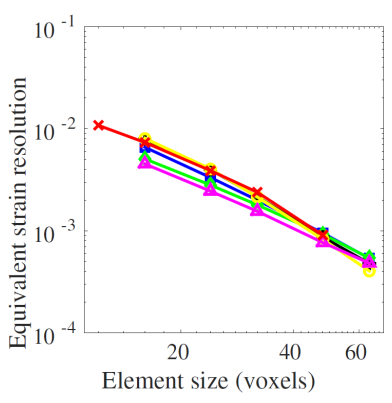

(b)

Figure 11: Standard displacement (a) and strain (b) resolutions as functions of the element size for bis cases

These results illustrate the classical compromise between spatial resolution and measurement uncertainties [39, 22, 21], which can be expressed by power law relationships $[39,23,30]$

$$
\sigma_{u}=\frac{A^{\alpha+1}}{\ell^{\alpha}} \quad \text { and } \quad \sigma_{\epsilon}=B \frac{\sigma_{u}}{\ell}
$$

where $A$ is a constant expressed in voxels, and $B$ a dimensionless quantity that depends on the way the strains are computed from the measured displacements. However, in the case of AA6061 T6, it can be noted that $B$ is not constant, but starts to decrease after reaching certain element sizes resulting in lower strain uncertainty levels compared with AA2198 T3 and AA2139 T8 grades for the element size $\ell=64$ voxels. This can be partly explained by fact that DVC benefits from reaching certain element sizes that overcome characteristic voids and intermetallic particles spacings (see Figure 6). 


\section{$3.2 \quad$ Effect of rigid body motion - rbm case}

For $r b m$ cases rigid body motions are applied between the two scans acquired. The experimental data available for this analysis are from AA2139 T3, AA2139 T8, AA2198 T3, AA2198 T8, AA6061 and cast iron samples (Table 1). Different levels and directions of rigid body motions have been applied in each case (see Section 2), the minimum level being for AA2198 T8 where only small motions in the S-direction occurred.

Figure 12 shows the displacement uncertainties for the three components and the mean level. For the samples that have experienced very large displacements along the L-direction (i.e., AA2139 T8, AA2198 T3, AA6061 T6), the corresponding displacement uncertainty degrades in comparison with the other displacement components. Displacements along the S-direction have also a marked effect (for sample AA6061 T6). 


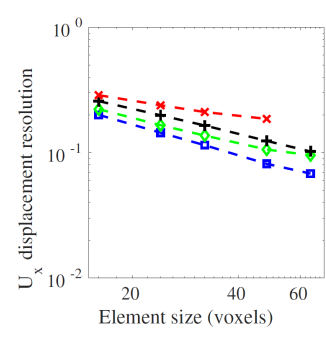

(a) $\mathrm{T}$

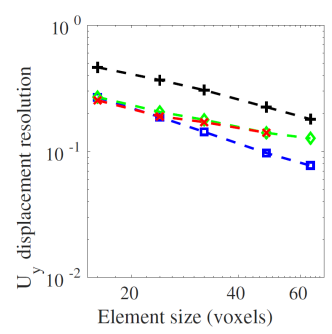

(b) $\mathrm{L}$

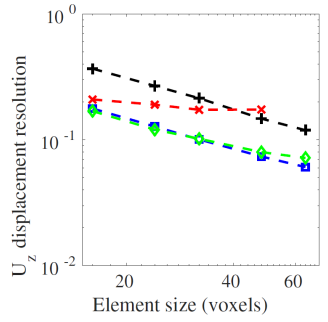

(c) $\mathrm{S}$

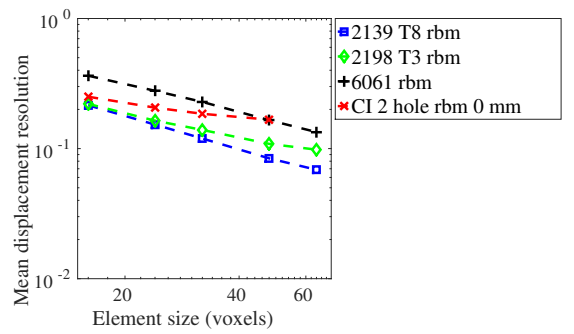

(d) mean

Figure 12: Standard displacement uncertainties as functions of the element size for $r b m$ cases

The fact that the reconstruction axis has moved with respect to microstructure features has a clear effect on the measurement uncertainties (when compared with bis cases), especially for the cases investigated herein, which are deemed very difficult since the volume fraction of "markers" is very low (Table 2). However, the overall trend for $r b m$ cases remains the same as in bis cases in terms of dependence with the element size. Along with previous statement, the AA2198 T8 microstructure showed the lowest uncertainties since the position of the reconstruction axis and consequently artifacts remained almost the same. Hence, this $r b m$ case is close to bis cases reported before.

Figure 13 shows the mean displacement uncertainties and the correspond- 
ing equivalent strain uncertainties. Even though the scatter in terms of strain uncertainties is higher than in the previous case (see Figure 11), the overall levels are very close and do not seem to be affected in a significant way by the amount of motion.

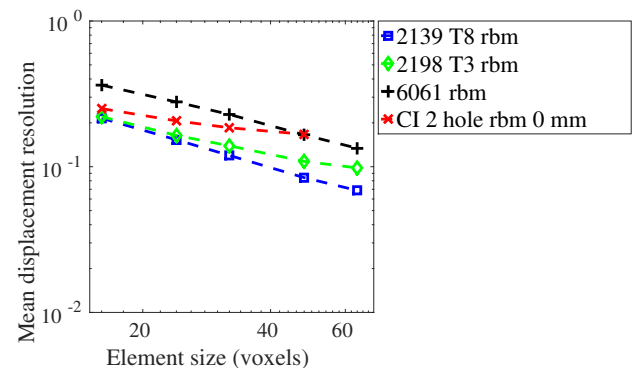

(a)

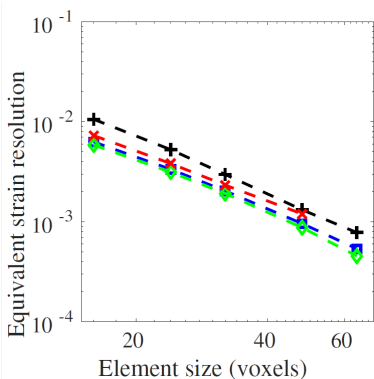

(b)

Figure 13: Standard displacement (a) and strain (b) uncertainties as functions of the element size for $r b m$ cases

\subsection{Comparison between bis and rbm cases}

In Figure 14 displacement and strain resolutions are explicitly compared for bis and rbm cases for the studied aluminum alloys and cast iron. After rigid body motions the reconstruction artifacts have changed their relative position with respect to the microstructural features, which is reflected in higher uncertainty levels for the $r b m$ cases. This effect is clearly observed on the mean displacement uncertainties in Figure 14(a). It can be noted that the microstructure with no voids and lower contrast (i.e., unfavorable gray level gradient for DVC registration) is more sensitive to rigid body motions. This effect is less pronounced for strain uncertainties (Figure 14(b)). 


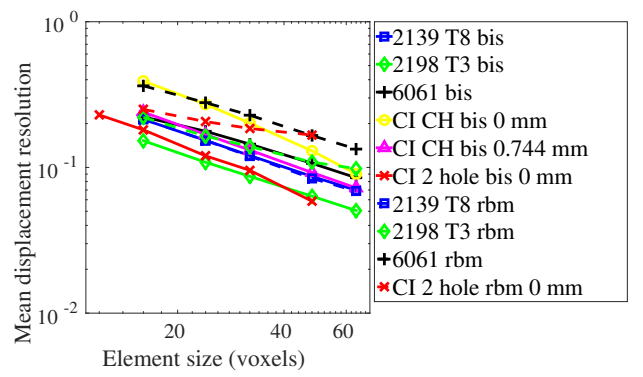

(a)

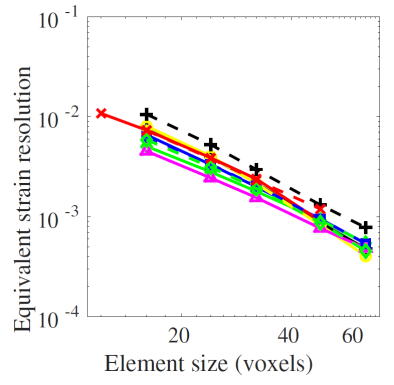

(b)

Figure 14: Standard displacement (a) and strain (b) resolutions as functions of the element size for bis and bis cases

These last results show that to fully evaluate measurement uncertainties it is better to move the sample before the second acquisition so that the rotation axis no longer coincides with that of the first reconstructed volume. Hence, in $r b m$ cases all the detrimental effects are accounted for and the reported uncertainty levels represent the stable limit above which mechanical signal is distinguished from the noise even for large displacement amplitudes.

Let us note that in some bis/rbm cases presented above the trends between displacement and strain resolutions differ significantly, e.g., for AA2198 T3 there is a significant gap between bis and rbm curves for displacement uncertainties while in the strain resolution graph the values almost coincide. This is probably due to the fact that measured rotations in the displacement uncertainty procedure are extracted globally on the ROI scale, while in the strain resolution procedure the Hencky strains exclude rotations more locally, i.e., at the $\mathrm{C} 8$ element level. 


\section{Influence of other parameters}

Three additional effects are analyzed with a more limited set of scans, namely, digitization level, monochromator and acquisition device. These parameters can be varied at the synchrotron facility where the scans were acquired.

\subsection{2 to 8 bit conversion - rescaling case}

When dealing with reconstructed volumes in tomography or laminography, post-processing issues arise because of the data size. That is why the reconstruction of 3D volumes from radiographs involves shift from the 32-bit floating point gray levels into 8-bit digitization. The parameters of this socalled rescaling procedure are chosen manually from the initial 32-bit floating point value histogram.

In the following two different rescaling parameters are utilized, thereby resulting in two different 8-bit reconstructed volumes obtained from the same initial radiographs. They will be distinguished by the suffix A or B in their names. Three combinations are analyzed to measure displacement fields, namely, reference scan A wrt. rbm scan A (denoted as "ref. scan A vs. rbm scan A" in Figure 15); reference scan B wrt. rbm scan B (denoted as "ref. scan B vs. rbm scan B"); reference scan A wrt. rbm scan B (denoted as "crossed").

Despite the difference of dynamic range the level of strain uncertainty remains virtually identical for the three combinations for any element size (Figure 15(b)). However, for the displacement uncertainty there is gap between "ref. scan B vs. rbm scan B" and the others revealing higher noise 
levels. This is confirmed by fact that in the "ref. scan B vs. rbm scan B" case, the calculation diverged for an element size $\ell=16$ voxels.

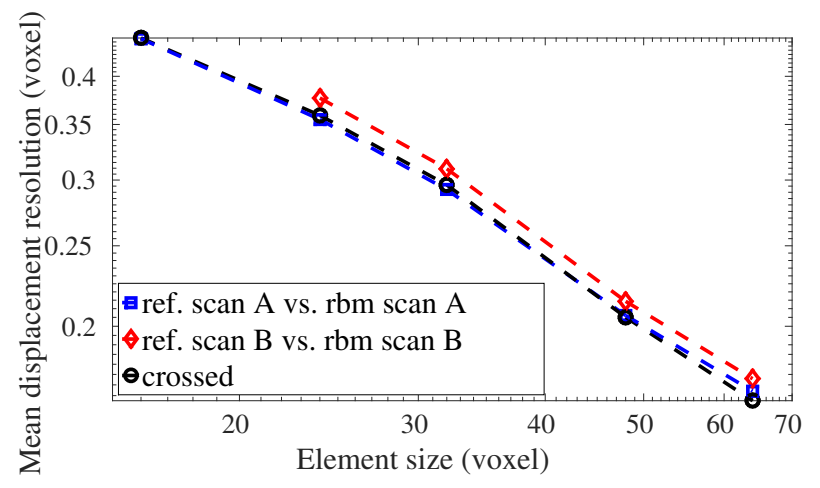

(a)

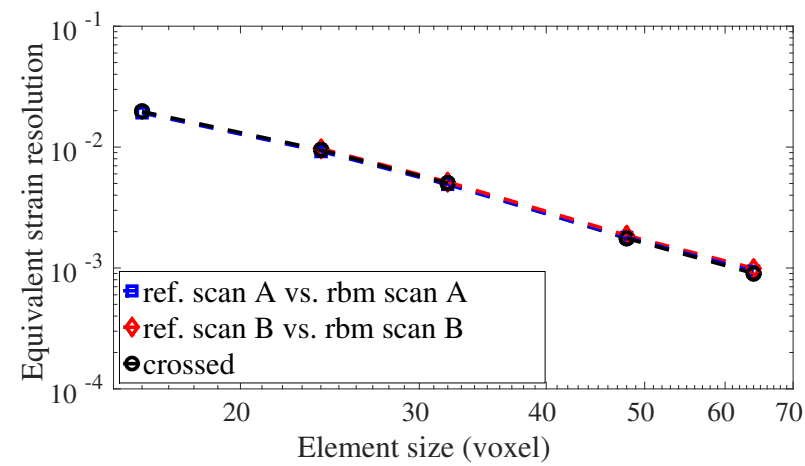

(b)

Figure 15: Standard displacement (a) and strain (b) resolutions as functions of the element size for rescaling cases. Two different rescaling parameters resulted in 2 different set of scans, namely: reference scan A and rbm scan A; reference scan B and rbm scan B. Three combinations are analyzed to measure displacement fields: reference scan A vs. $r b m$ scan A, reference scan B vs. $r b m$ scan B, and reference scan A vs. rbm scan B (crossed) 


\subsection{Beam properties}

The monochromator's main function is to extract from an incoming polychromatic X-ray beam a monochromatic beam of adjustable wavelength. The experimental data for AA2139 T3 and AA2198 T8 are obtained with a multilayer monochromator opposed to AA2139 T8 and AA2198 T3 scanned without monochromator. The multilayer monochromator was needed in order to limit the dose rate, beam hardening artifacts and high X-ray energies from the broad-band undulator source employed, which has rather low fundamental energies and multiple higher harmonics. The cross-section of the beam diffracted by the multilayer shows a characteristic modulated intensity pattern [36] stemming from long-range substrate and coating imperfections. These intensity modulations are prone to provoke ring artifacts after laminographic reconstruction. The AA2139 T8 and AA2198 T3 data were scanned without monochromator with radiation from a more recent short-period undulator providing mainly a single harmonic of sufficiently high energy and the higher harmonics are greatly reduced in intensity in comparison with the first harmonic. In that configuration, no monochromator is therefore needed anymore.

In Figure 16 both mean displacement and equivalent strain resolutions for the available data are shown. The data obtained with the monochromator are depicted with dashed lines and suffix "M" in the legend. There is no clear evidence of an influence of the monochromator for AA2198 alloy. As mentioned, AA2198 T8 rbm case is the result of small amounts of rigid body motion, hence these data can be understood as a bis case. This is 
illustrated in Figure 16(a) when compared to 2198 T3 bis data where a good agreement is found except for the larger element sizes. Hence, the monochromator presence may induce some additional uncertainty with longer characteristic wavelengths (i.e., rings), which results in a saturation of the uncertainty levels as shown in Figure 16(a) (see gap between "AA2198 T3 bis" and "AA2198 T8 rbm M" for large element sizes).

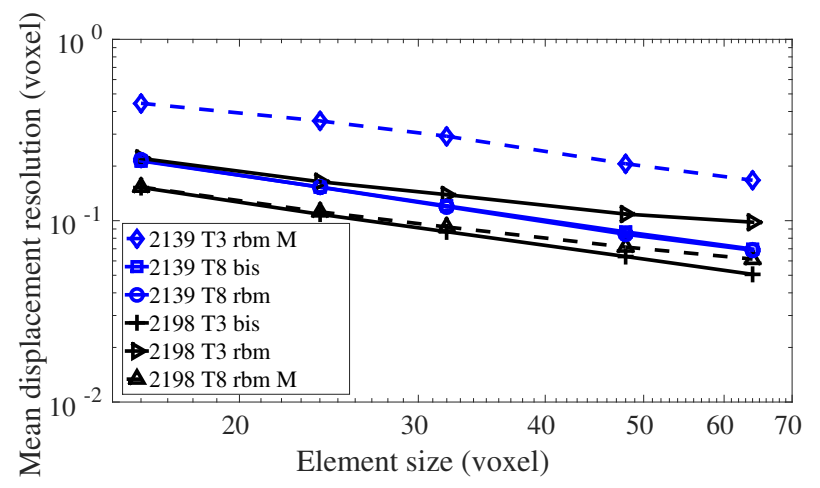

(a)

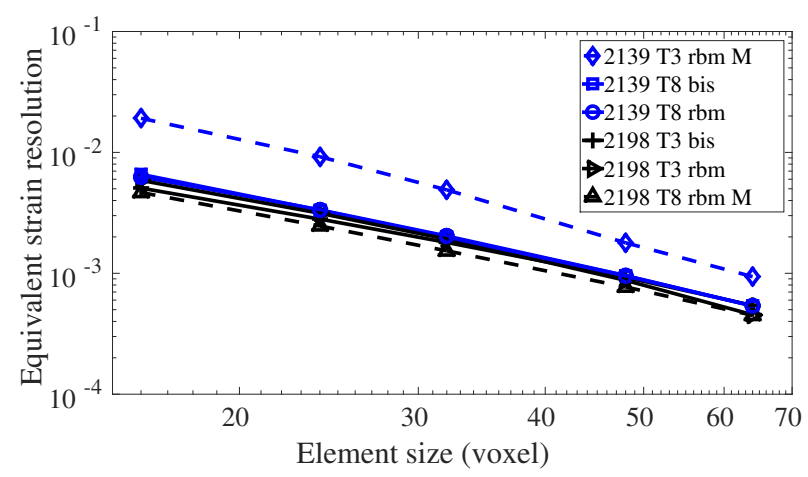

(b)

Figure 16: Standard displacement (a) and strain (b) resolutions as functions of the element size for different experimental setup

The fact that the "AA2139 T3 rbm M" case significantly deviates from "AA 2139 T8 rbm" should not be directly assigned to the monochromator 
influence. It is rather a consequence of different levels of applied rigid body motions and more importantly, the volume of the notch present in the reconstructed volume and ROI position with respect to it. Hence, the solution to probe this effect would be the existence of "AA2139 T8 bis M" data to compare it with its non-monochromator correspondent "AA2139 T8 bis" in conditions where there is no influence of rigid body motions.

\subsection{Detector}

The experimental data for AA2198 T8 material are obtained with two different types of detectors, namely the ESRF inhouse-designed FReLoN camera with $20 \mathrm{x}$ (i.e., $10 \mathrm{x}$ objective plus $2 \mathrm{x}$ eye-piece) optical magnification and the PCO.edge camera (PCO GmbH, Kelheim Germany) with 10x optical magnification (but both with similar scintillators converting the X-rays to visible light).

The FReLoN camera uses a sensor based on the charge-coupled device (CCD) principle while the PCO.edge camera uses a scientific CMOS sensor. Both converters have a dynamic range of about 14 bits but the full-well capacity of the PCO.edge is $\approx 5 \times$ lower so that less $\mathrm{X}$-ray photons are needed to saturate the detector. Consequently, also less photon statistics may be expected, resulting in noisier reconstructed images. To compensate for this effect to some extent, the number of X-ray projection images is increased from 2000 to 3600 images. Even with more images acquired the scan time still takes approximately only a third of the scan time with the CCD camera. 
The resolution of the CCD-based detector is $0.7 \mu \mathrm{m}$ while the CMOSbased one yields $0.65 \mu \mathrm{m}$. However, for the CMOS camera binning of $2 \times 2$ $\times 2$ voxels is performed in data postprocessing resulting in a final isometric voxel size of $1.3 \mu \mathrm{m}$. The CMOS camera shows slightly better results for the displacement resolution (see Figure $17(\mathrm{a})$ ) but this is also partly due to binning technique and larger physical size of the voxel for the CMOS camera. The equivalent strain resolution reveals similar values for both cases (Figure 17(b)), which underlines the same quality for both cameras in terms of final DVC measurement resolutions.

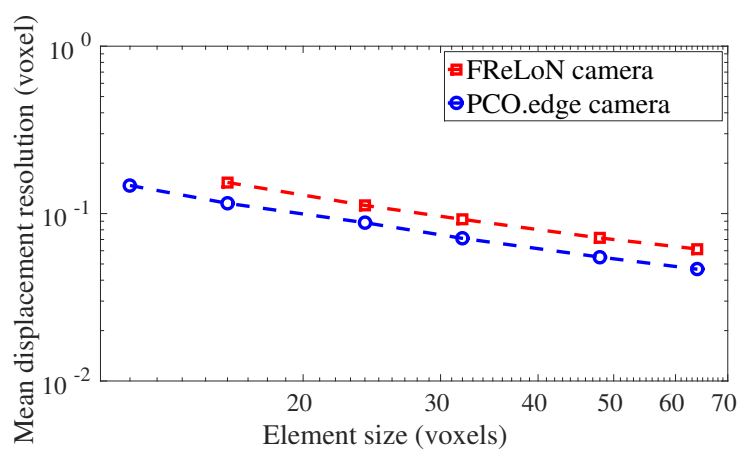

(a)

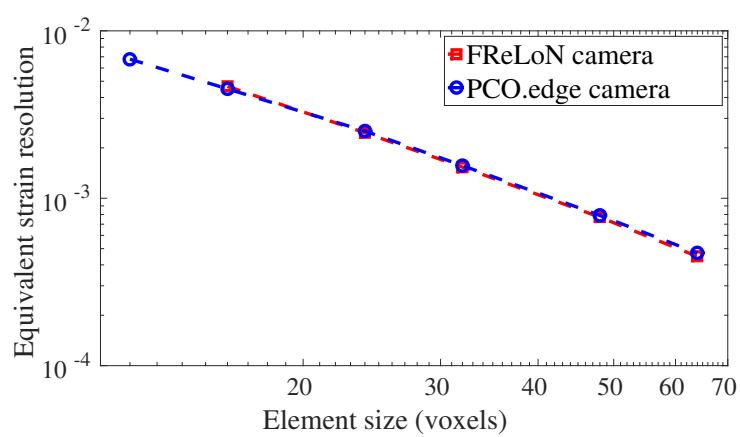

(b)

Figure 17: Standard displacement (a) and strain (b) resolutions as functions of the element size for different cameras used in the experiment 


\subsection{Discussion}

The effect of three additional parameters (i.e., rescaling, monochromator and detector) was studied in the present section. In all reported cases, it was found that their influence was not as important in comparison with the results discussed in Section 3.

\section{Conclusion}

Laminography is still a recent imaging technique [11, 13]. The use of reconstructed volumes for kinematic measurements has only started a few years ago $[40,30,46,9,32]$. Consequently, there is a need for metrological assessment of the measured displacement fields and the corresponding strain fields. The present paper aims at discussing ways of evaluating measurement uncertainties in this context. The procedures introduced herein are applied to aluminum alloys leading to weakly contrasted volumes when imaged by X-ray sources and cast iron with low contrast ferritic zones when imaged at high resolutions.

In the $\mathrm{Al}$ alloys studied, for any location of the region of interest, the uncertainty level is uniform for repeated scanning (bis) cases. However, when rigid body motions are applied between two acquisitions the ROI position close to the notch degrades the uncertainty level for AA2198 and AA6061 aluminum grades. The amount of initial voids or secondary particles is not necessarily sufficient to reach the lowest uncertainty level. In the present case, the AA2198 grade has the lowest volume fraction of the three aluminum alloys but also the lowest uncertainty. This result calls for caution 
and it is advisable to acquire at least two scans in the reference configuration, if possible by slightly moving the setup between the two acquisitions, to evaluate the practical displacement and strain resolutions of the studied material. Further, the strain resolution for cast iron virtually coincides with bis and rbm resolution levels for aluminum alloys, which supports the aforementioned limited influence of microstructure properties on the final measurement uncertainty levels in laminography.

By comparing the uncertainty levels measured with and without rigid body motions, for aluminum alloys and cast iron, it appears that the uncertainty mainly comes from the laminography technique and its associated artifacts $[15,48]$. The uncertainty levels are equal or higher with rigid body motions since some of the artifacts are not attached to the microstructure (like, e.g., phase-contrast edge enhancement) but intrinsic of the acquisition set-up (e.g., ring artifacts).

Last, let us note that the procedures discussed herein and applied to reconstructed volumes obtained by synchrotron laminography can also be applied to synchrotron or lab tomography. All these techniques require reconstruction steps from the raw data (i.e., radiographs) to the $3 \mathrm{D}$ volumes that induce additional artifacts depending on the equipment itself but also on the reconstruction algorithms.

\section{Acknowledgments}

The financial support of the Fédération Francilienne de Mécanique and Agence Nationale de la Recherche (ANR-14-CE07-0034-02 grant for COMIN- 
SIDE project) is gratefully acknowledged. Constellium C-Tech is acknowledged for aluminum alloys supply. M. Kuna, L. Zybell and M. Horn from IMFD, TU Freiberg, are thanked for cast iron supply and machining. We would like to acknowledge the European Synchrotron Radiation Facility for provision of beamtime at beamline ID15A and ID19 (experiments MA1006, MI1149, ME1366, MA2183).

\section{Appendices}

\section{Appendix A: Volumetric Correlation}

The DVC technique used herein is an extension of 2D global Digital Image Correlation $[5,19]$. The reconstructed volume is represented by a discrete scalar matrix of spatial coordinate $\mathbf{x}$ of (in this case 8-bit deep) gray levels determined by the microstructure absorption of X-rays. The principle of DVC consists of registering the gray levels $f$ in the reference configuration $\mathbf{x}$ and those of the deformed configuration $g$, which satisfy gray level conservation

$$
f(\mathbf{x})=g(\mathbf{x}+\mathbf{u}(\mathbf{x}))
$$

where $\mathbf{u}$ is the sought displacement field. However, due to acquisition noise, reconstruction artifacts [48] and the correlation procedure itself [22], ideal matching is not achieved in real examples. Consequently, the solution consists of minimizing the gray level residual

$$
\rho(\mathbf{x})=f(\mathbf{x})-g(\mathbf{x}+\mathbf{u}(\mathbf{x}))
$$


by considering its L2-norm with respect to kinematic unknowns. Since a global approach is used in this work [39], $C_{0}$ continuity over the Region of Interest (ROI) is prescribed to the solution and the global residual to be minimized reads

$$
\Phi_{c}^{2}=\sum_{R O I} \rho^{2}(\mathbf{x})
$$

The displacement field is parameterized by using a kinematic basis consisting of shape functions $\boldsymbol{\Psi}_{p}(\mathbf{x})$ and nodal displacements $u_{p}$

$$
\mathbf{u}(\mathbf{x})=\sum_{p} u_{p} \boldsymbol{\Psi}_{p}(\mathbf{x})
$$

Among a whole range of available fields, finite element shape functions are particularly attractive because of the link they provide between the measurement of the displacement field and numerical models [37, 16].

Thus, a weak formulation based on C8 finite elements with trilinear shape functions is chosen [39]. After successive linearizations and corrections the current system to be solved reads

$$
[\mathbf{M}]\{\delta \mathbf{u}\}=\{\mathbf{b}\}
$$

where

$$
M_{i j}=\sum_{R O I}\left(\boldsymbol{\nabla} f \cdot \boldsymbol{\Psi}_{i}\right)(\mathbf{x})\left(\boldsymbol{\nabla} f \cdot \boldsymbol{\Psi}_{j}\right)(\mathbf{x})
$$

represents the DVC matrix, while vector $\{\mathbf{b}\}$

$$
b_{i}=\sum_{R O I}(f(\mathbf{x})-\tilde{g}(\mathbf{x}))\left(\boldsymbol{\nabla} f \cdot \mathbf{\Psi}_{i}\right)(\mathbf{x})
$$

contains the current difference between the reference volume $f$ and the corrected deformed volume $\tilde{g}=g(\mathbf{x}+\tilde{\mathbf{u}}(\mathbf{x}))$, $\tilde{\mathbf{u}}$ being the current estimate of the displacement field. The global residual needs to decrease so that convergence 
is achieved, i.e., the displacement corrections $\{\delta \mathbf{u}\}$ become vanishingly small. In this form, the displacement field is regularized in the sense of a continuity requirement that is a priori assumed for the kinematic solution. Moreover, additional mechanical knowledge may be added to help convergence. This type of procedure is referred to as mechanical regularization [46]. It will not be used hereafter.

\section{Appendix B: Morphological characterization of Al alloys}

A priori analysis of contrast features (i.e., particles + voids) serves as quantification of material natural contrast, i.e., to predict the quality of volume gray level gradient that will serve as sensitivity for DVC registrations (see Equation (7)). Representative volumes with size $256 \times 256 \times 256$ voxels are extracted from ROIs used in DVC analyses (Figure 18).

Since it is case sensitive, slightly different threshold gray levels have been used to isolate voids and particles for the studied Al alloys. Before creating binary images all the original volumes are filtered in the open source software Fiji [41, 42, 43] by applying Gaussian blur with length of 1 voxel. 


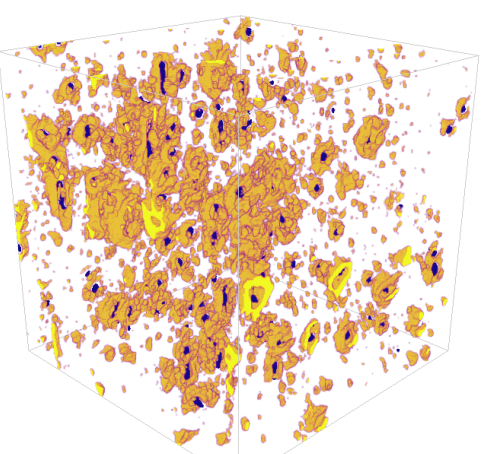

(a) AA2139 T3

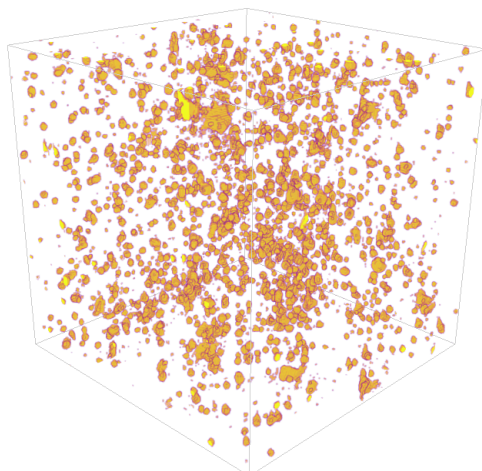

(c) AA2198 T3

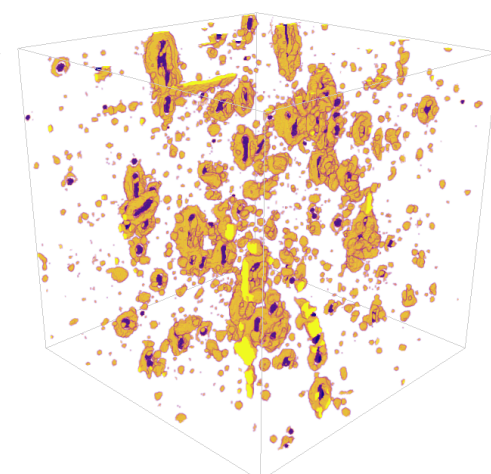

(b) AA2139 T8

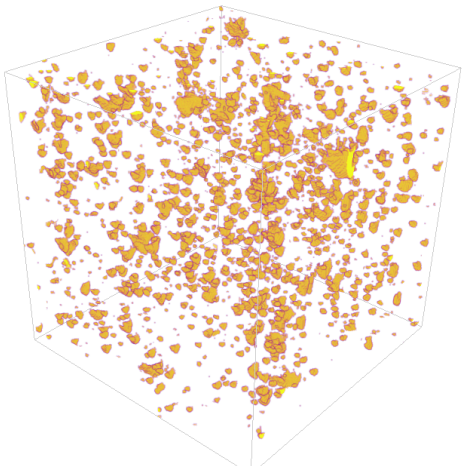

(d) AA2198 T8

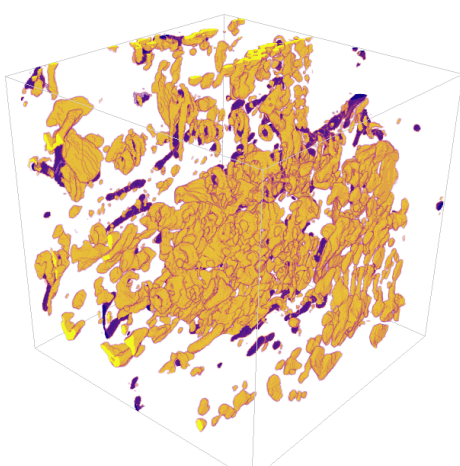

(e) AA6061 T6

Figure 18: Isometric isolated view of voids (blue) and particles (yellow) $(+$ edge-enhancement artifacts around voids due to so-called "phase contrast," an interference phenomenon due to Fresnel diffraction) in 3D reconstructed volumes $(256 \times 256 \times 256$ voxels $)$ of the studied aluminum alloys 
In AA2139 and AA6061 alloys the voids induce edge-enhancing phase contrast during the scanning procedure resulting in bright void boundaries (Figures 4(a), 4(b), 6 and 18(a-b,e)). These so-called cupping artifacts complicate particle separation based on intensity difference. The procedure shown in Figure 19 is followed to avoid particles over-counting induced by phase contrast. The voids are first isolated in a binary image. The particles and edge-enhancement artifacts are then separated from original volume. The two binary images are summed followed by merging the voids and corresponding edge-enhancement artifacts into single objects by Fiji functions (Process/Binary/Open). Hence, after these operations each void + edgeenhancement artifact carries single contrast having the same status as intermetallic particles. The counting of the objects is preformed by Fiji plugin $3 D$ object counter [6].

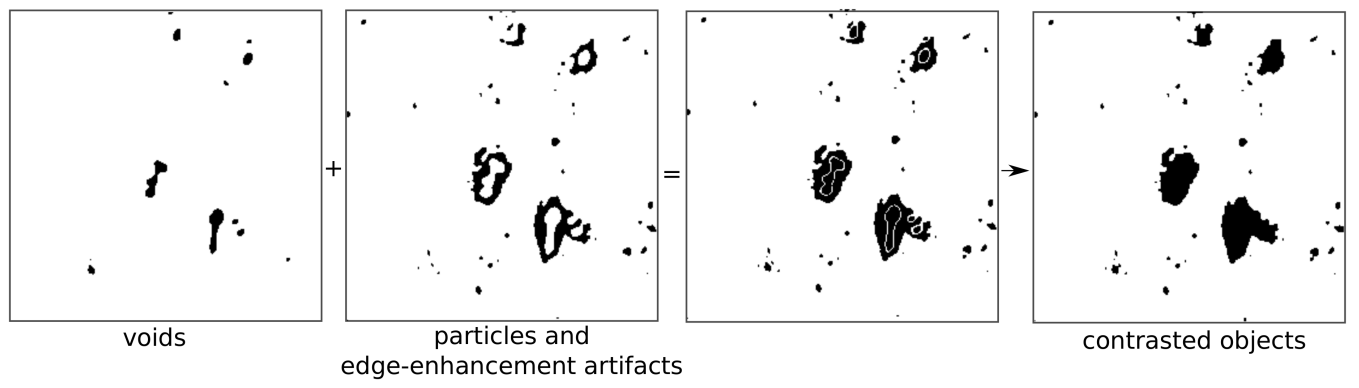

Figure 19: Procedure followed when isolating voids and particles for AA2139 and AA6061 alloys

The results, in terms of number of contrasted objects per element (where isotropic element size $\ell=32$ voxels), are listed in Table 2. Taking into account the low precision of the proposed method the results show there 
are no significant deviations (i.e., same order of magnitude) for any of the studied alloys. AA6061 T6 alloy has the lowest number of features available per element volume, but this needs to be taken with caution since AA6061 contains larger voids and particles (Figure 18(e)) where multiple elements can benefit from a single object, i.e., its boundary where gray level gradient is higher. This is outlined in the second row of Table 2 where the number of surface voxels (from all objects) per element volume is displayed.

Table 2: Number of features per element when the element size is $\ell=32$ voxels

\begin{tabular}{cccccc}
\hline Al alloy & AA2139 T3 & AA2139 T8 & AA2198 T3 & AA2198 T8 & AA6061 T6 \\
\hline features per el. & 3.5 & 2.6 & 4.7 & 3.3 & 1.0 \\
surface voxels per el. & 396 & 248 & 223 & 178 & 642
\end{tabular}

\section{Appendix C: Reconstruction artifact}

The sampling of the 3D Fourier domain of the region of interest is incomplete in laminography. Consequently the most outward voxels have only information from a limited range of viewing angles. This is illustrated in Figure 20 for three different sections along the sample thickness. The yellow line indicates the boundary of the region where the sample is in the field of view of the detector, which results in blurred reconstructed zones. Section B-B has artifacts due to "local" laminography, i.e., the projections are truncated with respect to the projection of the reconstruction volume, and this induces a directionality in the artifacts. In Section C-C let one note what is commonly called "reconstruction circle" in computed tomography. Outside these areas, the artifacts have a strong radial direction due to the missing information 
from tangential projection angles. For Section A-A, only the outer most voxels are concerned with such effects.
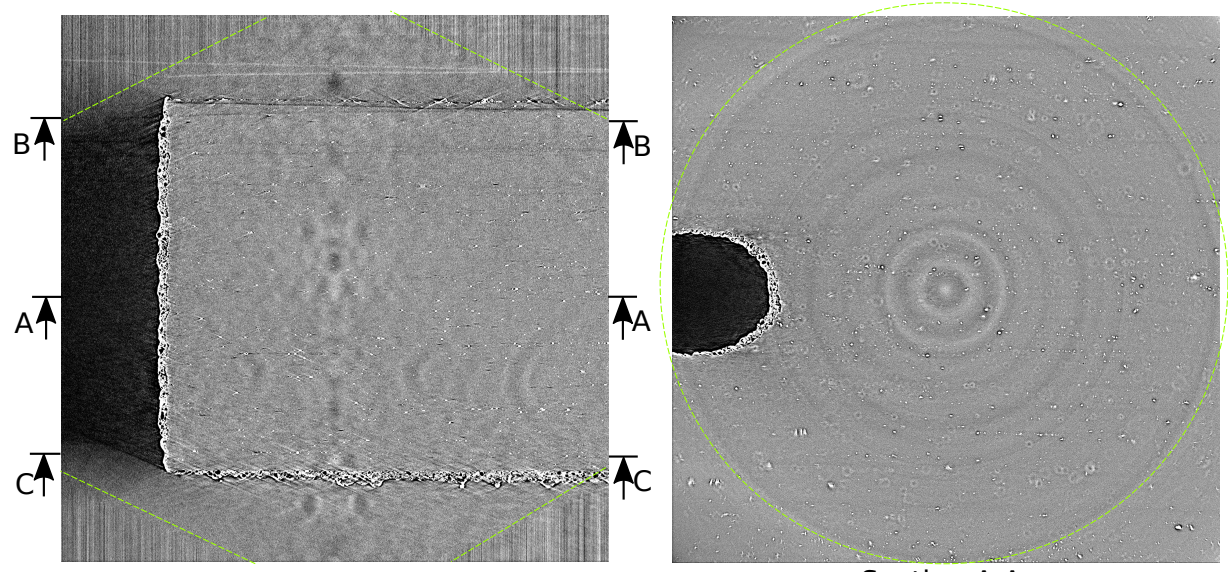

Section A-A

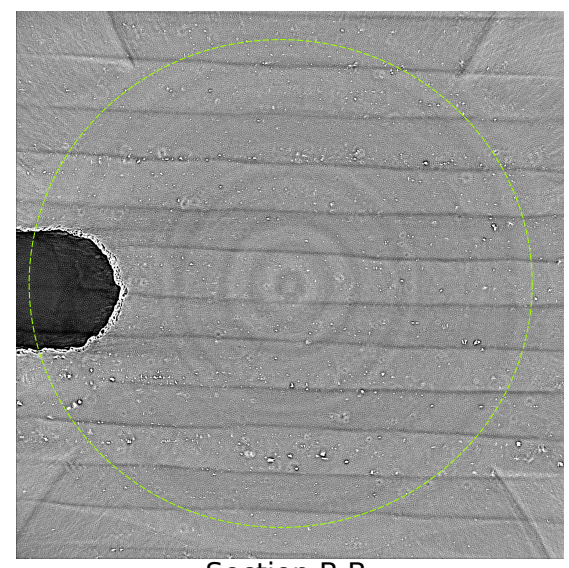

Section B-B

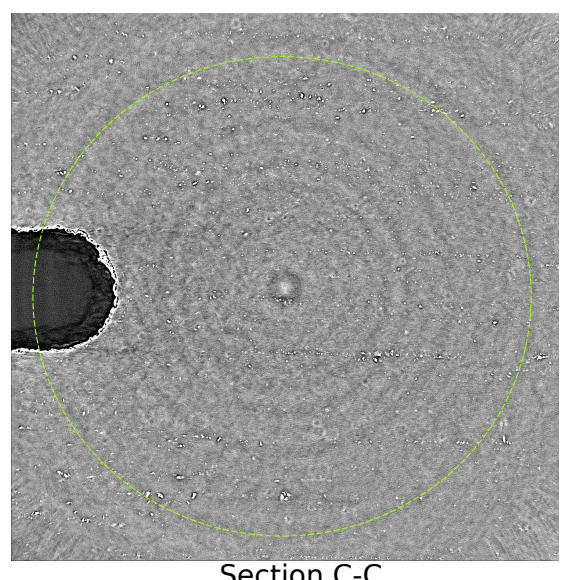

Figure 20: Reconstruction artifacts in laminography. The yellow line indicates the boundary of the region where the sample is inside the field of view of the detector for all projection directions.

\section{Appendix D: Morphological characterization of cast iron}

The analysis of contrasted features is conducted for cast iron by following the same procedure as for aluminum alloys described in Appendix B. The 
representative volume with size $256 \times 256 \times 256$ voxels is extracted from the ROI used in DVC analyses (Figure 21). Beside the isolated nodules one can notice a secondary void population in the ferritic matrix. The results, in terms of number of contrasted objects and surface voxels per element volume (isotropic element size $\ell=32$ voxels) are 1.5 and 458 respectively. These values are in the range of levels for aluminum alloys (Table 2).

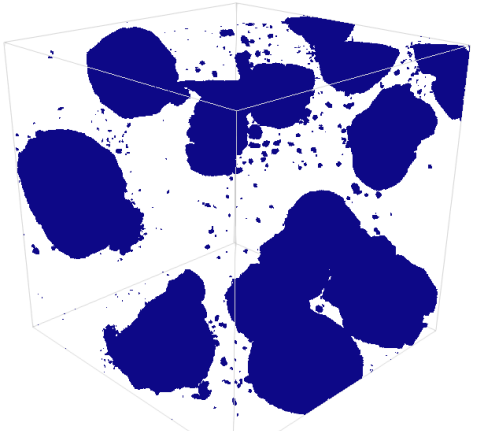

Figure 21: Isometric isolated view of nodules and secondary void population in $3 \mathrm{D}$ reconstructed volume $(256 \times 256 \times 256$ voxels $)$ of the studied cast iron 


\section{References}

[1] ISO/IEC guide 99-12:2007. International Vocabulary of Metrology - Basic and General Concepts and Associated Terms, VIM. International Organization for Standardization, Geneva (Switzerland), 2007.

[2] V. Altapova, L. Helfen, A. Myagotin, D. Hänschke, J. Moosmann, J. Gunneweg, and T. Baumbach, Phase contrast laminography based on Talbot interferometry. Optics Express, 20:6496-6508, 2012.

[3] J. Baruchel, J.Y. Buffière, E. Maire, P. Merle and G. Peix. X-Ray Tomography in Material Sciences. Hermes Science, Paris (France), 2000.

[4] A. Benoit, S. Guérard, B. Gillet, G. Guillot, F. Hild, D. Mitton, J. N. Périé and S. Roux. 3D analysis from micro-MRI during in situ compression on cancellous bone. Journal of Biomechanics, 42:2381-2386, 2009 .

[5] G. Besnard, F. Hild and S. Roux. "Finite-Element" Displacement Fields Analysis from Digital Images: Application to Portevin-Le Châtelier Bands. Experimental Mechanics, 46:789-803, 2006.

[6] S. Bolte and F. P. Cordelières. A guided tour into subcellular colocalization analysis in light microscopy. Journal of Microscopy, 224(3):213-232, 2006.

[7] J. Y. Buffière, E. Maire, J. Adrien, J. P. Masse and E. Boller. In Situ Experiments with X ray Tomography: an Attractive Tool for Experimental Mechanics. Experimental Mechanics, 50(3):289-305, 2010. 
[8] A. Buljac, M. Shakoor, J. Neggers, L. Helfen, M. Bernacki, P.-O. Bouchard, T. F. Morgeneyer and F. Hild. Numerical Validation Framework for Micromechanical Simulations based on Synchrotron 3D Imaging. Computational Mechanics, 59(3):419-441, 2017.

[9] A. Buljac, T. Taillandier-Thomas, T. F. Morgeneyer, L. Helfen, S. Roux and F. Hild. Slant strained band development during flat to slant crack transition in AA 2198 T8 sheet: in situ 3D measurements. International Journal of Fracture, 200(1):49-62, 2016.

[10] S. Gondrom, J. Zhou, M. Maisl, H. Reiter, M. Kröning and W. Arnold. X-ray computed laminography: an approach of computed tomography for applications with limited access. Nuclear Engineering and Design, 190(1):141-147, 1999.

[11] L. Helfen, T. Baumbach, P. Mikulík, D. Kiel, P. Pernot, P. Cloetens and J. Baruchel. High-resolution three-dimensional imaging of flat objects by synchrotron-radiation computed laminography. Applied Physics Letters, 86(7):071915, 2005.

[12] L. Helfen, A. Myagotin, P. Pernot, M. DiMichiel, P. Mikulík, A. Berthold and T. Baumbach. Investigation of hybrid pixel detector arrays by synchrotron-radiation imaging. Nuclear Instruments and Methods in Physics Research Section B, 563:163-166, 2006.

[13] L. Helfen, A. Myagotin, A. Rack, P. Pernot, P. Mikulík, M. Di Michiel and T. Baumbach. Synchrotron-radiation computed laminography for 
high-resolution three-dimensional imaging of flat devices. Physica Status Solidi (a), 204:2760-2765, 2007.

[14] L. Helfen, T. Baumbach, P. Cloetens and J. Baruchel. Phase-contrast and holographic computed laminography. Applied Physics Letters, 94, 104103, 2009.

[15] L. Helfen, A. Myagotin, P. Mikulík, P. Pernot, A. Voropaev, M. Elyyan, M. Di Michiel, J. Baruchel and T. Baumbach. On the implementation of computed laminography using synchrotron radiation. Review of Scientific Instruments, 82(063702), 2011.

[16] F. Hild, A. Bouterf, L. Chamoin, F. Mathieu, J. Neggers, F. Pled, Z. Tomičević and S. Roux. Toward 4D mechanical correlation. Advanced Modeling and Simulation in Engineering Sciences, 3(17), 2016.

[17] F. Hild, A. Fanget, J. Adrien, E. Maire and S. Roux. Three dimensional analysis of a tensile test on a propellant with digital volume correlation. Archives of Mechanics, 63(5-6):1-20, 2011.

[18] F. Hild, E. Maire, S. Roux and J. F. Witz. Three dimensional analysis of a compression test on stone wool. Acta Materialia, 57:3310-3320, 2009.

[19] F. Hild and S. Roux. Comparison of local and global approaches to digital image correlation. Experimental Mechanics, 52(9):1503-1519, 2012.

[20] A. Houssaye, F. Xu, L. Helfen, V. D. Buffrénil, T. Baumbach and P. Tafforeau. Three-dimensional pelvis and limb anatomy of the Cenomanian hind-limbed snake Eupodophis descouensi (Squamata, Ophidia) 
revealed by synchrotron-radiation computed laminography. Journal of Vertebrate Paleontology, 31(1):2-7, 2011.

[21] H. Leclerc, J.N. Périé, F. Hild and S. Roux. Digital volume correlation: What are the limits to the spatial resolution? Mechanics $\&$ Industry, 13:361-371, 2012.

[22] H. Leclerc, J.N. Périé, S. Roux and F. Hild. Voxel-scale digital volume correlation. Experimental Mechanics, 51(4):479-490, 2011.

[23] N. Limodin, J. Réthoré, J. Adrien, J. Y. Buffière, F. Hild and S. Roux. Analysis and artifact correction for volume correlation measurements using tomographic images from a laboratory x-ray source. Experimental Mechanics, 51(6):959-970, 2011.

[24] N. Limodin, J. Réthoré, J. Y. Buffière, A. Gravouil, F. Hild and S. Roux. Crack closure and stress intensity factor measurements in nodular graphite cast iron using three-dimensional correlation of laboratory X-ray microtomography images. Acta Materialia, 57(14):40904101, 2009.

[25] L. Liu and E. Morgan. Accuracy and precision of digital volume correlation in quantifying displacements and strains in trabecular bone. Journal of Biomechanics, 40:3516-3520, 2007.

[26] K. Madi, G. Tozzi, Q. H. Zhang, J. Tong, A. Cossey, A. Au, D. Hollis and F. Hild. Computation of full-field displacements in a scaffold implant using digital volume correlation and finite element analysis. Medical Engineering \& Physics, 35(9):1298-1312, 2013. 
[27] E. Maire, J. Y. Buffière, L. Salvo, J. J. Blandin, W. Ludwig and J. M. Létang. On the Application of X-ray Microtomography in the Field of Materials Science. Advanced Engineering Materials, 3(8):539-546, 2001.

[28] V. Maurel, L. Helfen, F. N'Guyen, A. Koster, M. Di Michiel, T. Baumbach and T. F. Morgeneyer. Three-dimensional investigation of thermal barrier coatings by synchrotron-radiation computed laminography. Scripta Materialia, 66(7):471-474, 2012.

[29] T. Morgeneyer, T. Taillandier-Thomas, L. Helfen, T. Baumbach, I. Sinclair, S. Roux and F. Hild. In situ 3D observation of early strain localisation during failure of thin al alloy (2198) sheet. Acta Materialia, 69:78-91, 2014.

[30] T.F. Morgeneyer, L. Helfen, H. Mubarak and F. Hild. 3D digital volume correlation of synchrotron radiation laminography images of ductile crack initiation: An initial feasibility study. Experimental Mechanics, 53(4):543-556, 2013.

[31] T.F. Morgeneyer, L. Helfen, I. Sinclair, H. Proudhon, F. Xu and T. Baumbach. Ductile crack initiation and propagation assessed via in situ synchrotron radiation computed laminography. Scripta Materialia, 65:1010-1013, 2011.

[32] T.F. Morgeneyer, T. Taillandier-Thomas, A. Buljac, L. Helfen and F. Hild. On strain and damage interactions during tearing: 3D in situ measurements and simulations for a ductile alloy (AA2139-T3). Journal of the Mechanics and Physics of Solids, 96:550-571, 2016. 
[33] T. Weitkamp, P. Tafforeau, E. Boller, P. Cloetens, J.-P. Valade, P. Bernard, F. Peyrin, W. Ludwig, L. Helfen and J. Baruchel. Parallelbeam imaging at the ESRF beamline ID19: current status and plans for the future. AIP Conference Proceedings, R. Garrett, I. Gentle, K. Nugent and S. Wilkins (edts.), 1234:83-86, 2010.

[34] M. Di Michiel, J. M. Merino, D. Fernandez-Carreiras, T. Buslaps, V. Honkimäki, P. Falus, T. Martins, O. Svensson, Fast microtomography using high energy synchrotron radiation", Review of Scientific Instruments, 76:043702, 2005.

[35] A. Myagotin, A. Voropaev, L. Helfen, D. Hänschke and T. Baumbach. Efficient Volume Reconstruction for Parallel-Beam Computed Laminography by Filtered Backprojection on Multi-Core Clusters. IEEE Transactions on Image Processing, 22(12):5348-5361, 2013.

[36] A. Rack, T. Weitkamp, M. Riotte, D. Grigoriev, T. Rack, L. Helfen, T. Baumbach, R. Dietsch, T. Holz, M. Krämer, F. Siewert, M. Meduna, P. Cloetens and E. Ziegler. Comparative study of multilayers used in monochromators for synchrotron-based coherent hard X-ray imaging. Journal of Synchrotron Radiation, 17:496-510, 2010.

[37] J. Rannou, N. Limodin, J. Réthoré, A. Gravouil, W. Ludwig, M. C. Baïetto, J. Y. Buffière, A. Combescure, F. Hild and S. Roux. Three dimensional experimental and numerical multiscale analysis of a fatigue crack. Computer Methods in Applied Mechanics and Engineering, 199:1307-1325, 2010. 
[38] S. Roux and F. Hild. Stress intensity factor measurements from digital image correlation: post-processing and integrated approaches. International Journal of Fracture, 140(1-4):141-157, 2006.

[39] S. Roux, F. Hild, P. Viot and D. Bernard. Three dimensional image correlation from X-ray computed tomography of solid foam. Composites Part A, 39(8):1253-1265, 2008.

[40] S. Roux, T. Taillandier-Thomas, A. Bouterf, H. Leclerc, T. F. Morgeneyer and F. Hild. Digital volume correlation from tomographic images: Results and challenges. In IUTAM symposium on Advances of Optical Methods in Experimental Mechanics, 2012.

[41] J. Schindelin, I. Arganda-Carreras, E. Frise, V. Kaynig, M. Longair, T. Pietzsch, S. Preibisch, C. Rueden, S. Saalfeld, B. Schmid, J. Y. Tinevez, D. J. White, V. Hartenstein, K. Eliceiri, P. Tomancak and A. Cardona. Fiji: an open-source platform for biological-image analysis. Nature Methods, 9(7):676-682, 2012.

[42] J. Schindelin, C. T. Rueden, M. C. Hiner and K. W. Eliceiri. The imagej ecosystem: An open platform for biomedical image analysis. Molecular Reproduction and Development, 82(7-8):518-529, 2015.

[43] C. A. Schneider, W. S. Rasband and K. W. Eliceiri. NIH Image to ImageJ: 25 years of image analysis. Nature Methods, 9(7):671-675, 2012.

[44] M. Shakoor, A. Buljac, J. Neggers, F. Hild, T. F. Morgeneyer, L. Helfen, M. Bernacki and P.-O. Bouchard. On the choice of boundary conditions 
for micromechanical simulations based on 3D imaging. International Journal of Solids and Structures, 112:83-96, 2017.

[45] Y. Shen, T. F. Morgeneyer, J. Garnier, L. Allais, L. Helfen and J. Crépin. Three-dimensional quantitative in situ study of crack initiation and propagation in aa6061 aluminum alloy sheets via synchrotron laminography and finite-element simulations. Acta Materialia, 61(7):2571-2582, 2013 .

[46] T. Taillandier-Thomas, S. Roux, T. F. Morgeneyer and F. Hild. Localized strain field measurement on laminography data with mechanical regularization. Nuclear Instruments and Methods in Physics Research Section B, (324):70-79, 2014.

[47] A. Weck and D. S. Wilkinson. Experimental investigation of void coalescence in metallic sheets containing laser drilled holes. Acta Materialia, 56(8):1774-1784, 2008.

[48] F. Xu, L. Helfen, T. Baumbach and H. Suhonen. Comparison of image quality in computed laminography and tomography. Optics Express, 20:794-806, 2012. 